\title{
18. CALCAREOUS NANNOFOSSILS ACROSS CRETACEOUS/TERTIARY BOUNDARY AT SITE 752, EASTERN INDIAN OCEAN ${ }^{1}$
}

\author{
James J. Pospichal ${ }^{2}$
}

\begin{abstract}
A biostratigraphically complete Cretaceous/Tertiary boundary was recovered during Ocean Drilling Program Leg 121 . The boundary, cored in ODP Hole 752B on Broken Ridge, is the most expanded deep-sea section yet recovered by ODP/DSDP. The initial Danian subzone, CP1a, spans nearly $5 \mathrm{~m}$ and the underlying uppermost Maestrichtian Nephrolithus frequens Zone extends $50 \mathrm{~m}$ below the boundary. The paleolatitude of Broken Ridge at Cretaceous/Tertiary time is estimated at $50^{\circ}-55^{\circ} \mathrm{S}$ which includes this site among the latest in a series of complete or near complete high southern latitude Cretaceous/Tertiary boundary sections recovered by ODP (Leg 113 Site 690 and Leg 119 Site 738).

The boundary at Site 752 lies at the base of a thick $(6-6.5 \mathrm{~m})$ volcanic ash unit composed of multiple ash layers which overlies indurated Maestrichtian chalks. Magnetostratigraphy indicates that the boundary lies within Subchron 29R, which is the case for all other known complete sections for which the polarity has been determined. Anomalous abundances of the trace element iridium are present at the boundary. A second iridium peak, $80 \mathrm{~cm}$ above the boundary, corresponds to an increase in redeposited Cretaceous nannofossils.

The nannofossil succession is similar to that found at previously studied austral high-latitude ODP drill sites with few differences due to the more northerly location of this site. Individual nannofossil species were counted and placed into three categories. A plot of the percent abundance of Cretaceous, Tertiary, and "survivor" groups illustrates the rapid replacement of the Cretaceous nannoflora by "survivor" forms beginning at the boundary and the dominance of this latter group through the initial Danian biozone. This "survivor" or opportunistic assemblage is then rapidly replaced by newly evolved Tertiary taxa. The assemblage of the uppermost Maestrichtian is biased toward dissolution-resistant forms such as Micula decussata. In those few intervals where preservation is good, the dissolution susceptible species, Prediscosphaera stoveri, is more prevalent and overall diversity of the assemblage is higher. The "survivor" assemblage is dominated by Zygodiscus sigmoides and Thoracosphaera. The Tertiary assemblage consists of rare Biantholithus sparsus, the first of this group to appear. It is followed several meters upsection by Cruciplacolithus primus. Cruciplacolithus tenuis and small Prinsius spp. dominate the assemblage beginning at about $5 \mathrm{~m}$ above the boundary.
\end{abstract}

\section{INTRODUCTION}

Recent deep-sea drilling in the southern Atlantic and Indian oceans by Ocean Drilling Program (ODP) Legs 113, 119, and 121 has produced several complete or nearly complete Cretaceous/Tertiary boundary sections. Previous to these legs, little was known about the Cretaceous/Tertiary boundary in the high southern latitudes. Leg 113 (Site 690) on Maud Rise in the Weddell Sea cored the first continuous Cretaceous/Tertiary section at the extreme high-latitude of $65^{\circ} \mathrm{S}$ (Fig. 1A). The heavily bioturbated muddy nannofossil chalks of the Maestrichtian-Danian interval contain nannofossil assemblages similar to those of boreal sections but are unique in many ways (Pospichal and Wise, 1990a). Recorded at Site 690 was the first observation of a bloom of Hornibrookina just above the Cretaceous/Tertiary boundary within Okada and Bukry (1980) Subzone CP1a. An overwhelming dominance of uppermost Maestrichtian assemblages by Prediscosphaera stoveri was also noted. A similar or possibly identical form was noted in the lower latitude sections of El Kef and Brazos River by Jiang and Gartner (1986).

Until the southern Indian Ocean legs it was not known whether the characteristic Maestrichtian-Danian assemblages of Site 690 were unique and, therefore, exclusive to the Atlantic sector of the Southern Ocean. In the Indian Ocean, a laminated, continuous Cretaceous/Tertiary sequence was cored on the southern tip of the

\footnotetext{
'Weissel, J., Peirce, J., Taylor, E., Alt, J., et al., 1991. Proc. ODP, Sci. Results, 121: College Station, TX (Ocean Drilling Program). U.S.A.

${ }^{2}$ Department of Geology, Florida State University, Tallahassee, FL 32306,
}

Kerguelen Plateau (Site $738,63^{\circ}$ S) (Fig. 1A). Nannofossil assemblages there are similar to the high-latitude South Atlantic (Thierstein et al., in press; Wei and Thierstein, in press; Wei and Pospichal, in press). A Hornibrookina bloom occurs in the upper portion of Subzone CP1a, as well as abundant Prediscosphaera stoveri in the uppermost Maestrichtian assemblage.

These initial results from Sites 690 and 738 suggested a general nannofossil biogeographic pattern for the high austral latitudes for Cretaceous/Tertiary time. This pattern is further exemplified by results from Legs 120 and 121 . Legs 120 and 121 drilled sites on the central portion of the Kerguelen Plateau and Broken Ridge, respectively, the latter having constituted the northernmost part of the plateau until it rifted and spread north during the middle Eocene (Fig. 1).

A hiatus encompassing Subzone CP1a is present at Site 750 $\left(58^{\circ} \mathrm{S}\right)$ (Schlich, Wise, et al., 1989). However, abundant Hornibrookina was recorded in the lowermost Tertiary samples of Subzone CP1b (Ehrendorfer and Aubry, in press), which suggests that a bloom may also have occurred at this site. In addition, abundant Prediscosphaera stoveri were also noted in the uppermost Maestrichtian sediments (Watkins, in press).

During Leg 121, a biostratigraphically complete Cretaceous/Tertiary boundary was cored at Site 752 (paleolatitude $50^{\circ}-55^{\circ} \mathrm{S}$ ) on Broken Ridge, which completed the northern end of the Kerguelen Plateau transect (Fig. 1A). Two strong iridium peaks were noted by Michel et al. (this volume) and Schuraytz et al. (this volume), one at the Cretaceous/Tertiary boundary and another about $80 \mathrm{~cm}$ above it. The core containing the boundary is composed of numerous drilling "biscuits" produced by the rotary coring process (Fig. 2). As all the nannofossil zones are present, it is difficult to tell how much section, if any, may be 
A
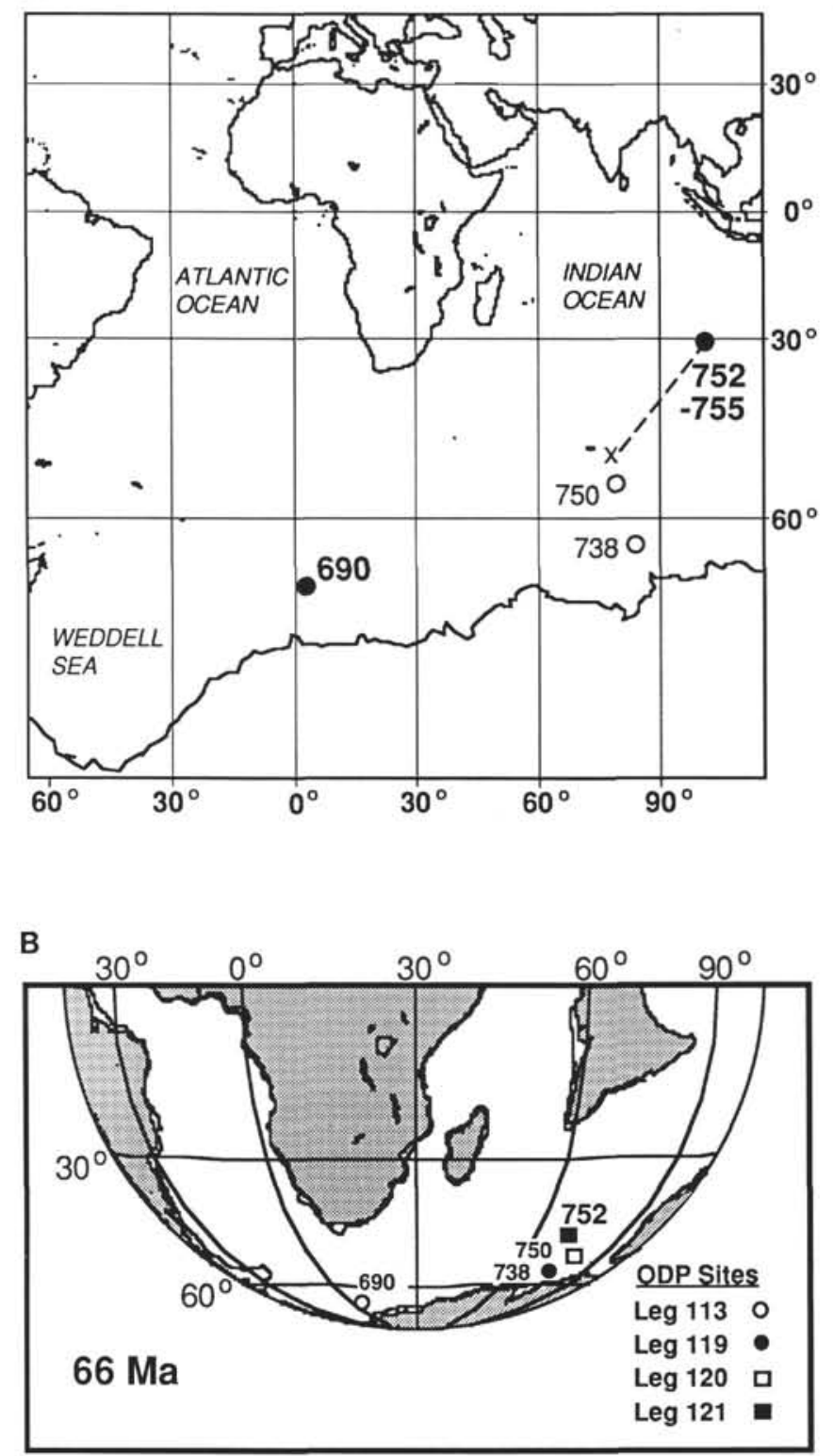

Figure 1. A. Location map of ODP Leg 121 Broken Ridge Sites 752-755, Leg 113 Site 690 , Leg 120 Site 750, and Leg 119 Site 738. The pre-rift location of Site 752 on the northern end of the Kerguelen Plateau is marked X. B. The estimated locations of these sites at $66 \mathrm{Ma}$ (reconstructions from C. R. Scotese and C. R. Denham, TERRA MOBILIS ${ }^{\mathrm{tm}}$ ).

missing between these "biscuits." Nevertheless, this section is the most expanded deep-sea sequence yet recovered by ODP/DSDP. At this site, a continuous supply of volcanic ash through the late Maestrichtian and early Danian produced sedimentation rates slightly higher than that normally found at other deep-sea sites. This, despite the drop in $\mathrm{CaCO}_{3}$ flux associated with the collapse in surface productivity at the end of the Maestrichtian, resulted in an expanded boundary interval.

This paper summarizes a quantitative study of calcareous nannofossils in closely-spaced samples from the Cretaceous/Tertiary boundary interval at Site 752. The study delimits the northern extent in the Indian Ocean of the austral biogeographic province delineated from Sites 690,738 , and 750 . The proximity of these sites at Cretaceous/Tertiary boundary time is illustrated in Figure 1B which shows a rough approximation of the paleopositions of these sites for $66 \mathrm{Ma}$.

\section{BROKEN RIDGE}

Broken Ridge is a shallow-water volcanic platform that formed the northernmost portion of the Kerguelen-Heard Plateau during Aptian-Cenomanian times. The separation and present location of Broken Ridge are due to rifting and seafloor spreading, which began about anomaly 18 time (middle Eocene) and continued to the present. Broken Ridge has moved north about $20^{\circ}$ of latitude as a part of the Indo-Australian plate, while its conjugate, the Kerguelen-Heard Plateau, as a part of the Antarctic plate, has moved very little. Throughout its history, Broken Ridge has been at fairly shallow depths collecting pelagic carbonate sediments, which comprise an expanded and nearly complete sedimentary section. Sediment accumulation was interrupted to a measurable degree on only two occasions. During the rifting process, rapid uplift (middle Eocene) exposed parts of Broken Ridge above sea level and subsequent wave-base erosion produced the distinctive angular unconformity shown in the seismic profile of Figure 3. In addition, a middle to upper Oligocene disconformity is present in the sections at Sites 752 and 754 .

Four closely spaced sites (Fig. 3) were drilled at Broken Ridge to address questions concerning lithospheric response to extension (Peirce, Weissel, et al., 1989). The Eocene-Recent sequence above the angular unconformity at Broken Ridge is described as a winnowed, stark white to pale brown nannofossil ooze. The sediments immediately overlying the unconformity, of which little was recovered, are composed of upper Eocene unconsolidated sands and gravels indicative of a shallow-marine origin.

At Sites 752 and 754, cored at water depths of 1097 and 1076 $\mathrm{m}$, respectively, continuous, highly expanded Maestrichtianlower Eocene ash-rich nannofossil chalks were recovered from below the angular unconformity. Ash layers are abundant in the Maestrichtian and decrease upsection. Chert and porcellanite are common in the lower Maestrichtian and Paleocene units. At Site 755 , in a water depth of $1069 \mathrm{~m}, 143 \mathrm{~m}$ of Turonian through Santonian ash with limestone was cored; however, the majority or all of the Campanian lies within a 460-m uncored section between the lowermost sediments recovered at Site 754 and the youngest Cretaceous sediments of Site 755 (Fig. 3). Also, about $190 \mathrm{~m}$ of lower and middle Eocene chalks remained undrilled between the bottom of Hole 753A and the top of the chalks underlying the unconformity at Hole 752B. The lithologies from Sites 752-755 are summarized in Figure 3.

\section{Cretaceous/Tertiary Boundary - Site $\mathbf{7 5 2}$}

The Cretaceous/Tertiary boundary is located within a unit of well-indurated, ash-rich chalk in Hole 752B (Figs. 2 and 4). The boundary is placed at Sample 121-752B-11R-3, 94-95 cm, or at approximately $358.75 \mathrm{~m}$ below seafloor (mbsf), well within Subchron 29R (Gee et al., this volume). The first occurrence (FO) of the Tertiary calcareous nannofossil Biantholithus sparsus was noted in the section at $72-73 \mathrm{~cm}$ and the last occurrence (LO) of Cretaceous planktonic foraminifers in the section at $94-95 \mathrm{~cm}$ (Fig. 4). The boundary lies within a sequence of ash, chert, and chalk, which immediately underlies a $6-\mathrm{m}$ to $6.5-\mathrm{m}$ ash layer. The lithology of the boundary interval as shown in Figure 4 is described in detail in Peirce, Weissel, et al. (1989). The ash layer present between 90 and $95 \mathrm{~cm}$ overlies light gray, mottled, and well-indurated uppermost Maestrichtian chalk containing the last Cretaceous foraminifers. Immediately above this, in the interval from 85 to $90 \mathrm{~cm}$, is a light-colored chalk with soft-sediment deformation structures. The overlying interval between 75 and 85 $\mathrm{cm}$ is composed of gray chert and porcellanite, which underlies 


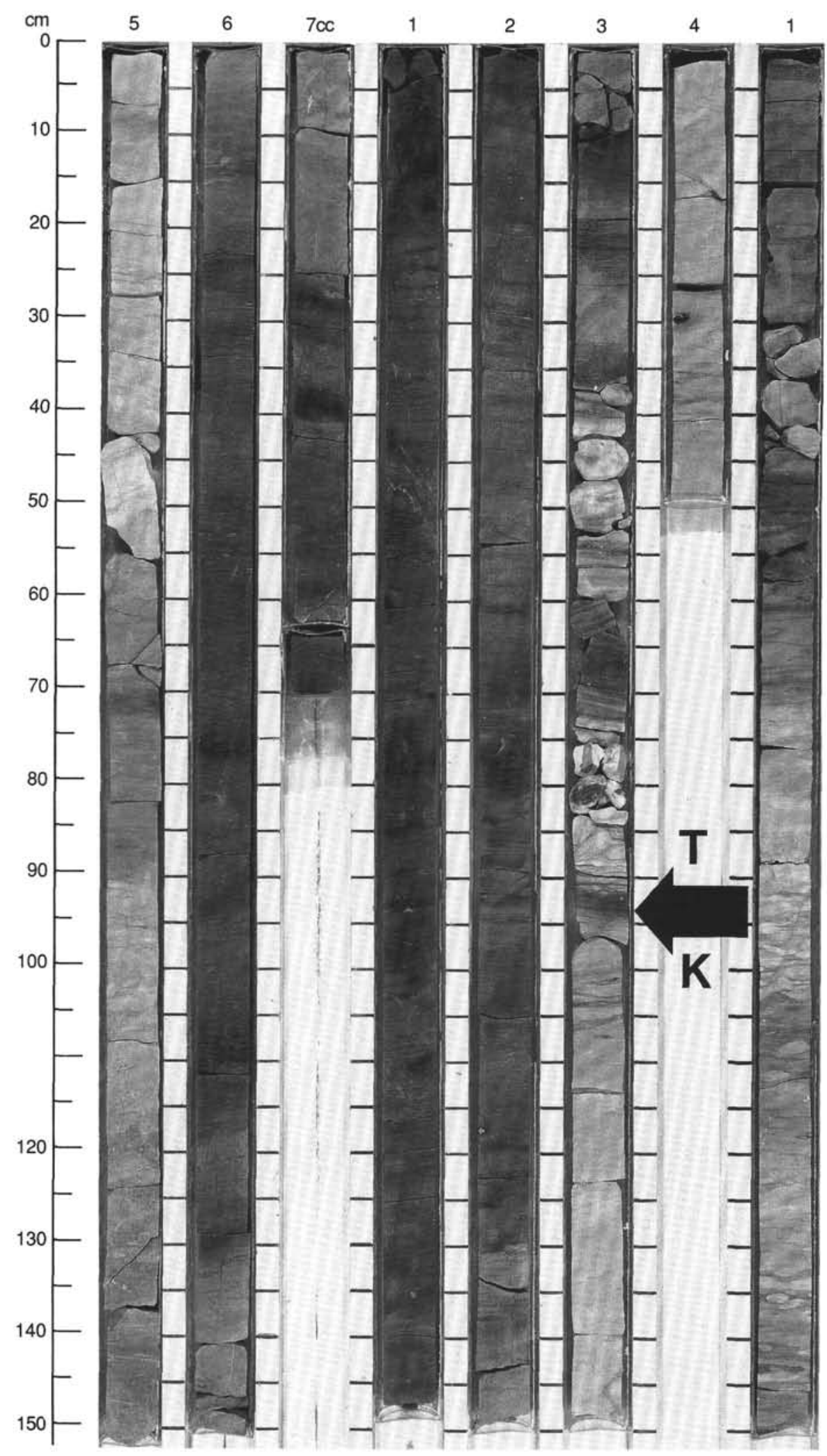

Figure 2. The Cretaceous/Tertiaary boundary is located at Sample 121-752B-11R-3, $94-95 \mathrm{~cm}$. The dark sediment is volcanic ash and the light-colored sediment is chalk. 


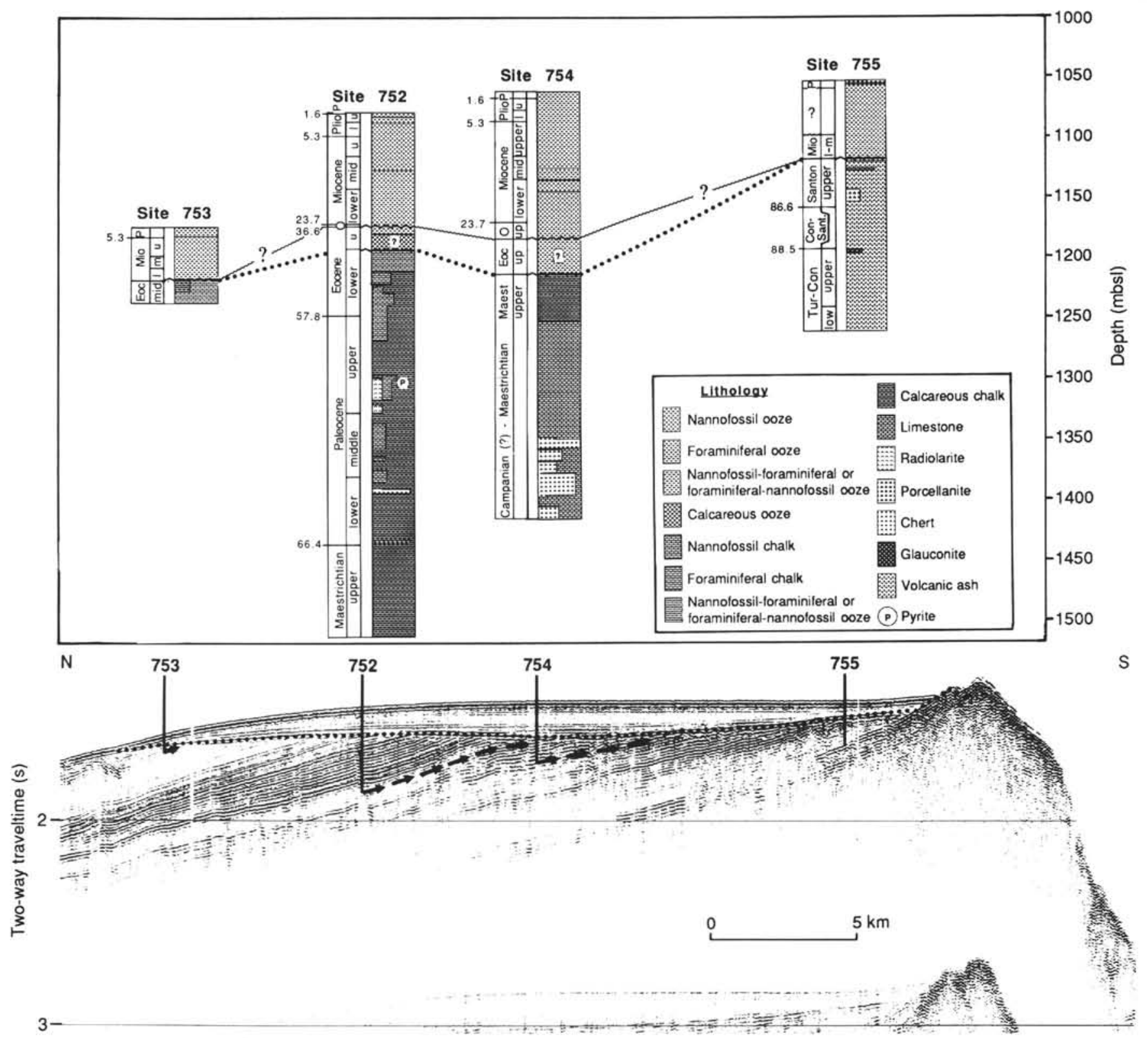

Figure 3. Seismic stratigraphy and lithostratigraphy at Broken Ridge showing locations and Sites 752-755. The arrows represent the upward continuation of the deepest level penetrated at Sites 752-754, showing the amount of section recovered and any stratigraphic overlap at these sites. The dotted line represents the middle Eocene hiatus, and the wavy line, the Oligocene hiatus. The two hiatuses converge at Sites 753 and 755 , but the question marks indicate that the position where they coalesce across Broken Ridge is unresolved.

chalk containing B. sparsus that grades upward into ash to about $60 \mathrm{~cm}$. This is overlain by $25 \mathrm{~cm}$ of chalk, which lies just below an approximately 6-m-thick ash layer.

The entire Cretaceous/Tertiary sequence described here, and especially the cherty interval, is composed of numerous drilling "biscuits" produced by twisting and breaking during the rotary coring process (Fig. 4). Thus, all interpretations through this interval must be made with caution because much sediment can be missing from between these "biscuits." Recovery in Core 121$752 \mathrm{~B}-11 \mathrm{R}$ was only $52 \%$ of the $9.6 \mathrm{~m}$ cored. However, logging data (Wilkinson, this volume) suggest that most of the overlying $6 \mathrm{~m}$ of ash was recovered, and recovery in the overlying Core $121-752 \mathrm{~B}-10 \mathrm{R}$ was $100 \%$.

\section{Ash and $\mathrm{CaCO}_{3}$}

An interesting feature of the Broken Ridge Cretaceous and Paleogene section is the large amount of ash. Mass accumulation rates on Broken Ridge for volcanic ash and calcium carbonate are shown in Figure 5 with detailed descriptions of these sedimentary components given in Peirce, Weissel, et al. (1989) and Rea et al. (1990). Ash flux was high during the Turonian through Santonian, which is exemplified by the recovery of $143 \mathrm{~m}$ of dark greenishgray to black ash with limestone and glauconite in Hole $755 \mathrm{~A}$ (Fig. 3). Ash is also a major component of the section immediately overlying the Cretaceous/Tertiary boundary as indicated by the presence of a $6-6.5-\mathrm{m}$ ash layer. Magnetic susceptibility data 


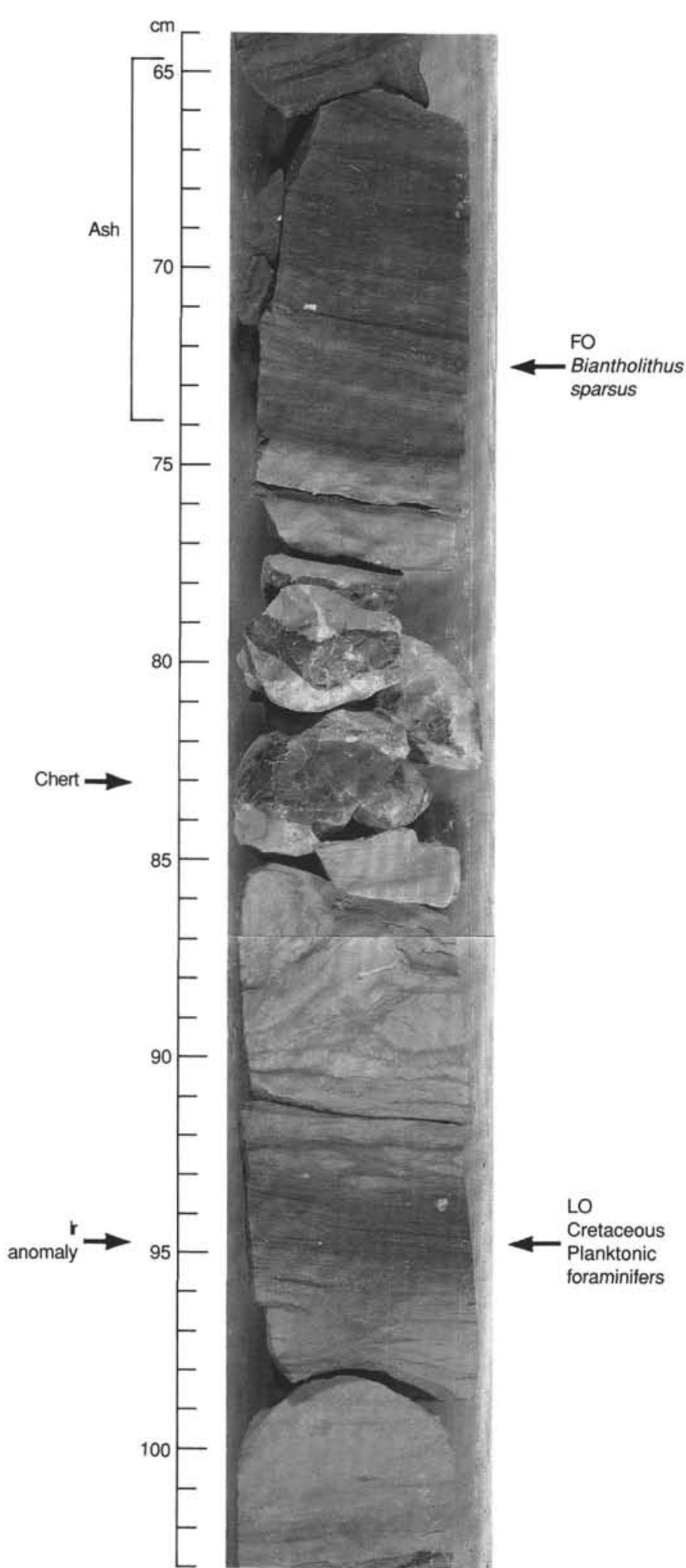

Figure 4. Enlarged view of the Cretaceous/Tertiary boundary (Section 121752B-11R-3, 63-103 cm). The FO of Biantholithus sparsus and the LO of Cretaceous foraminifers are indicated, along with the location of the iridium anomaly, chert, and ash layers.

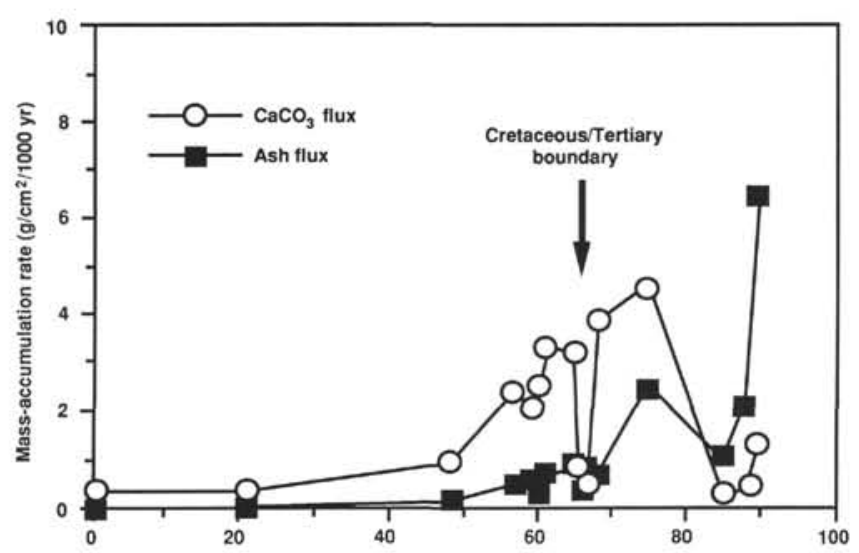

Figure 5. Mass-accumulation rates of ash and calcium carbonate on Broken Ridge from the middle Cretaceous to present (from Peirce, Weissel, et al., 1989). Note the major decline in $\mathrm{CaCO}_{3}$ at Cretaceous/Tertiary boundary.

suggest that this ash unit is the result of multiple ash falls and not a single event (Peirce, Weissel, et al., 1989). In addition, the ash unit does not represent a sudden influx of ash, but is the result of normal ash accumulation in the absence of calcium carbonate sedimentation. This is illustrated in Figure 5 and by the analysis of the calcium carbonate and volcanic ash flux data as reported in Peirce, Weissel, et al. (1989) and summarized in the following.

Ash flux values decline through the Turonian-Santonian inter-

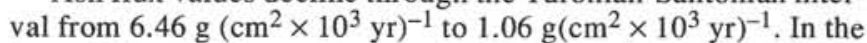

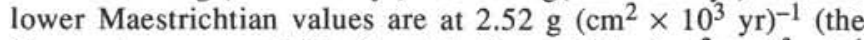

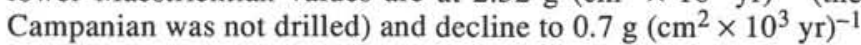
in the uppermost Maestrichtian. At the same time, calcium carbonate flux values in the Turonian through Santonian are as low as $0.36 \mathrm{~g}\left(\mathrm{~cm}^{2} \times 10^{3} \mathrm{yr}\right)^{-1}$ in the upper Santonian and jump to values up to $4.66 \mathrm{~g}\left(\mathrm{~cm}^{2} \times 10^{3} \mathrm{yr}\right)^{-1}$ in the Maestrichtian. Across the Maestrichtian/Danian boundary, ash flux increases only slightly from $0.7 \mathrm{~g}\left(\mathrm{~cm}^{2} \times 10^{3} \mathrm{yr}\right)^{-1}$ in the uppermost Maestrichtian to $0.9 \mathrm{~g}\left(\mathrm{~cm}^{2} \times 10^{3} \mathrm{yr}\right)^{-1}$ in the basal Danian. The calcium carbonate flux drops by an order of magnitude, from 3.9 to $0.4 \mathrm{~g}\left(\mathrm{~cm}^{2} \times 10^{3} \mathrm{yr}\right)$. Opal accumulation also declines across the boundary from $0.19 \mathrm{~g}\left(\mathrm{~cm}^{2} \times 10^{3} \mathrm{yr}\right)^{-1}$ to $0.07 \mathrm{~g}\left(\mathrm{~cm}^{2} \times 10^{3}\right.$ yr $)^{-1}$.

Thus, the significant change in the sedimentary component across the Cretaceous/Tertiary boundary is in the accumulation of the biogenic component as ash flux increases only slightly. The sharp reduction in carbonate flux represents a major drop in productivity in the early Danian. This period of low productivity (low carbonate flux) lasted at least $1.0 \mathrm{~m} . \mathrm{y}$, possibly more before returning to more "normal" values (Fig. 5). This duration is consistent with that suggested by the carbon isotope analyses of the deep-sea Cretaceous/Tertiary section of Site 577 (North Pacific) by Zachos et al. (1989). However, it is longer than that proposed by Stott and Kennett (1989) who suggest a duration of $250,000 \mathrm{yr}$ as indicated by carbon isotope data of Site 690 (Weddell Sea).

\section{CALCAREOUS NANNOFOSSILS}

\section{Previous Work}

Initial work on calcareous nannofossils in Cretaceous/Tertiary boundary sections was conducted by Bramlette and Martini (1964). Important stratigraphic studies were later done by Edwards (1966) in New Zealand, Hay and Mohler (1967) in France, 


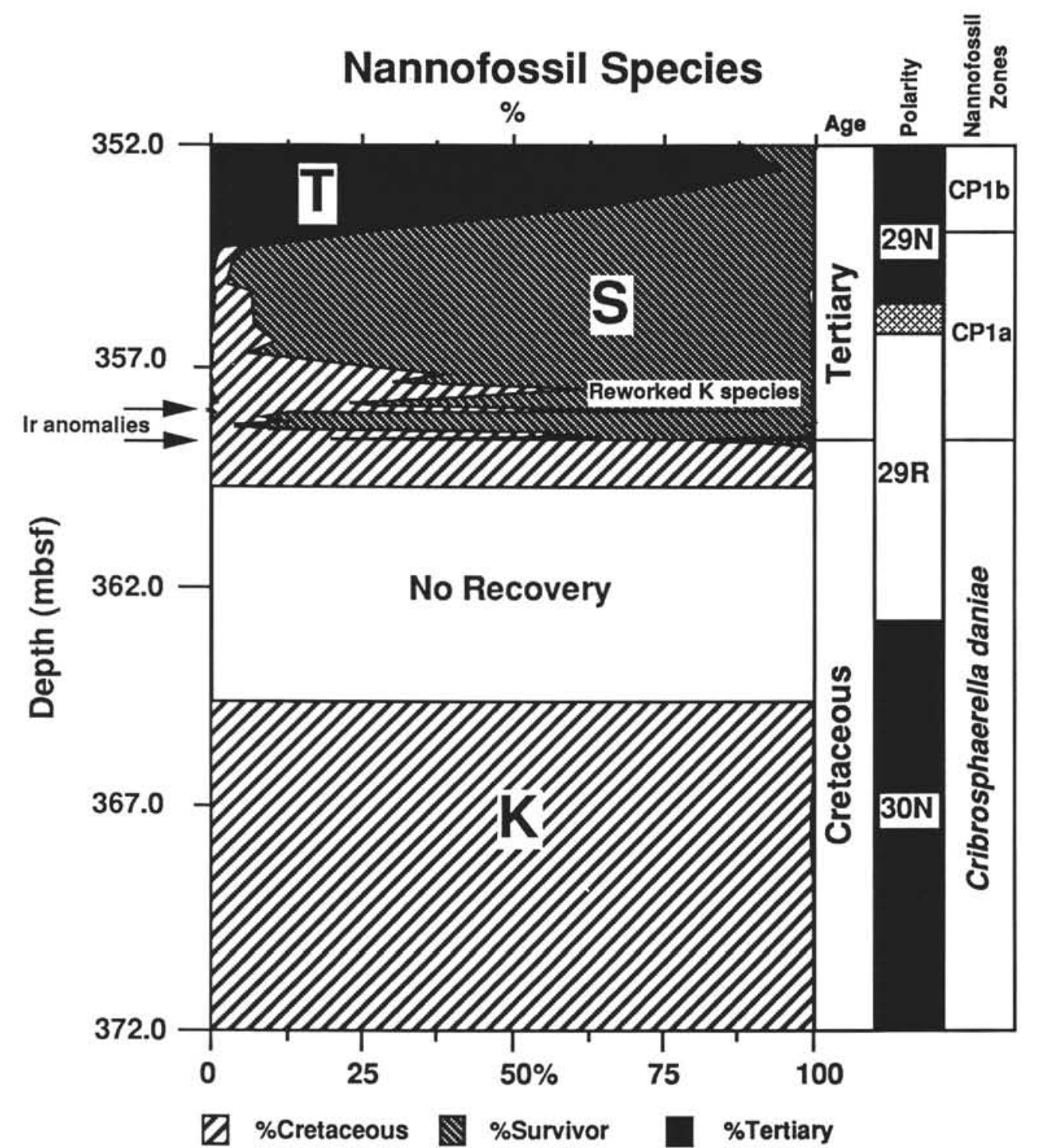

Figure 6. Percent abundance of Cretaceous (K) vs. Tertiary (T) vs. "survivor" (S) nannofossil species across the Cretaceous/Tertiary boundary interval of $352.0-372.0 \mathrm{mbsf}$ of ODP Hole 752B. Magnetostratigraphy is shown correlated with nannofossil stratigraphy. Stippled area in the polarity column denotes margin of error for the Subchron 29N/29R boundary.

Perch-Nielsen (1969) in Denmark, Worsley (1974) in Alabama, Romein (1977) in Spain, Monechi (1977) in Italy, and PerchNielsen (1981b) in Tunisia. A formerly high-latitude sequence recovered by DSDP drilling in the Southern Hemisphere was described by Edwards (1973). Thierstein (1981), Perch-Nielsen et al. (1982), and Smit and Romein (1985) published comprehensive reviews with information on calcareous nannofossils and planktonic foraminifers compiled from a geographically wide range of Cretaceous/Tertiary boundary sites.

Many of the studies previously mentioned are primarily works of a qualitative nature. Quantitative methods for which this was patterned were done by Percival and Fischer (1977), Thierstein and Okada (1979), and Jiang and Gartner (1986). Quantification and statistical analysis permit a more accurate assessment of the assemblage turnover across the boundary, which can then be easily used for comparison on a regional or global basis. However, regardless of the accuracy of these methods, problems still remain when comparing quantitative analyses by different workers as individual methods and species concepts vary to a certain extent.

\section{Methods}

Toothpick samples were taken aboard ship at $1-\mathrm{cm}$ increments across the boundary interval and at additional intervals where deemed necessary. Routine $3-\mathrm{cm}^{3}$ samples were taken at $4-\mathrm{cm}$ increments across the boundary and at successively longer intervals above and below the boundary.

Smear slides of raw sediment were analyzed under the light microscope at $1560 \times$, and at least 500 specimens per sample were counted. The count of 500 is designed to ensure that infrequent taxa are included. Most samples were examined beyond this count, however, to uncover any additional species present and to assure that no stratigraphic information was lost. Such is the case with Biantholithus sparsus, which was not observed during the counting of Sample 121-752B-11R-3, 72-73 cm, but was ob- 
served during routine shipboard examination of the same slide. Specimens not observed while counting were not considered statistically and are indicated in the range chart (Table 1) by an X. Samples 121-752B-20R-3, 92-93 cm, to 121-752B-10R-5, 1-2 $\mathrm{cm}$, where counts of only 300 were made, were examined as a part of biogeographic study (Pospichal, unpubl. data), but are also included in Table 1. Also, in the case of samples in Section $121-752 \mathrm{~B}-11 \mathrm{R}-3$ around the Cretaceous/Tertiary boundary at 56, $50,76,80,84$, and $88 \mathrm{~cm}$, nannofossils are extremely rare, and the count of 500 could not be reached within a reasonable amount of time. In such instances, as many specimens as possible were counted, and percent abundances of individuals were still calculated as shown in Table 1. However, the numbers from these intervals would be considered statistically insufficient. These nannofossil-poor intervals correspond with the previously described cherts and porcellanites.

All specimens counted were placed in one of three categories: Tertiary, Cretaceous, or "survivor." The procedure is similar to that used by Thierstein and Okada (1979) for DSDP Site 384, Percival and Fischer (1977) for the Zumayan section of Spain, and Pospichal and Wise (1990a) for ODP Site 690. The percentages of these three groups were calculated and plotted in Figure 6 and listed in Table 1 . Individual abundance counts are included in Table 1 with percent abundances of selected taxa plotted in Figure 7. Overall abundance and preservation of nannofossils as listed in Table 1 was estimated using the following criteria.

For abundance estimated at $1560 \mathrm{x}: \mathrm{V}=$ very abundant $(>10$ specimens per field of view); $A=$ abundant (6-10 specimens per field of view); $\mathrm{C}=$ common ( $1-5$ specimens per field of view); $\mathrm{F}$ $=$ few (one specimen per $2-10$ fields of view) $\mathrm{R}=$ rare (one specimen per $>10$ fields of view).

Preservation is estimated as follows: $\mathrm{G}=\operatorname{good}$ (little or no overgrowth and/or dissolution; species identification not hindered); $\mathrm{M}=$ moderate (some overgrowth and/or dissolution; species identification still possible); $\mathrm{P}=$ poor (abundant overgrowth and/or dissolution; species identification severely hindered but often possible).

For this study, the following species were combined in order to facilitate counting: Arkhangelskiella cymbiformis and A. specillata, Gartnerago spp., Thoracosphaera spp., Cyclagelosphaera alta and $C$. reinhardtii, Coccolithus cavus and $C$. pelagicus, and Prinsius tenuiculum and $P$. dimorphosus.

\section{Counting Thoracosphaera}

Counting Thoracosphaera can be extremely difficult because specimens of Thoracosphaera usually occur as fragments. Whole specimens are rare. Unfortunately, this does not allow great accuracy when counting. The procedure used here for counting thoracosphaerid fragments is similar to that of Jiang and Gartner (1986). Pieces considered to be about one-fourth to one-third of a specimen were counted and added up to one. Half specimens were tallied and fragments considered to be three-fourths or greater were counted as one specimen. The final piece count was then rounded up where necessary. For example, if the half fragments totaled 3 ( 1.5 specimens), this was rounded up to 2 specimens in the final total given in Table 1.

\section{Cretaceous/Tertiary Boundary Biostratigraphy}

Various authors of calcareous nannofossil biostratigraphic schemes use different criteria for defining the Cretaceous/Tertiary boundary. The last occurrence of Cretaceous taxa is commonly used (Martini, 1971; Okada and Bukry, 1980; Sissingh, 1977) or, in some cases, the FO of Biantholithus sparsus (Perch-Nielsen, 1979a; Pospichal and Wise, 1990a). van Heck and Prins (1987) used the sudden change in nannofossil assemblages to delimit the Cretaceous/Tertiary boundary. All of these methods for defining the boundary are not without problems. Bioturbation and reworking can lead to a misplacement of the boundary by more than $1 \mathrm{~m}$ using any of these criteria (Pospichal et al., 1990). Fortunately, lithologic, geochemical, and color changes usually associated with the Cretaceous/Tertiary boundary assist in the biostratigraphic delineation of that horizon (Smit and Romein, 1985). The biostratigraphy of the boundary interval correlated with the magnetostratigraphy (Gee et al., this volume) is shown in Figure 6 . The zonation scheme of Okada and Bukry (1980) is employed for the basal Danian and the Cribrosphaerella daniae Subzone of Pospichal and Wise (1990b) for the uppermost Maestrichtian.

At Site 752 , boundary placement using lithologic criteria was not so straightforward. The interval contains numerous dark green ash layers within light-colored chalks of the uppermost Maestrichtian underlying the thick basal Danian ash unit (Figs. 2 and 4). Thus, there is no single color change but an alternation of colors. No characteristic boundary clay is present; instead, chert and porcellanite occur. The section is further complicated by the poor condition of the core produced by the drilling process. Therefore, by visual inspection the boundary could be placed within an interval between 70 and $95 \mathrm{~cm}$ (Fig. 4). A likely location, using lithologic criteria, is at $80 \mathrm{~cm}$, or the interval corresponding to the dark chert layer. This is between the shipboard nannofossil biostratigraphic boundary and the planktonic foraminifer boundary. An iridium anomaly, however, is present at 93-94 cm (Michel et al., this volume), and this is more consistent with onshore placement of the boundary by nannofossil analysis in this study.

The FO of Biantholithus sparsus at $72 \mathrm{~cm}$ in Section 121 $752 \mathrm{~B}-11 \mathrm{R}-3$ and the LO of Cretaceous planktonic foraminifer species at $94 \mathrm{~cm}$ was used to delineate the Cretaceous/Tertiary boundary aboard ship (Peirce, Weissel, et al., 1989). The quantitative study allows for a more precise location of the boundary in terms of calcareous nannofossils. Using the criteria of van Heck and Prins (1987) of assemblage change, the boundary is located below the first noted $B$. sparsus and probably lies between 80 and $95 \mathrm{~cm}$ in Section 121-752B-11R-3. As shown in Table 1, the change in abundance of "survivor" forms begins to occur at 92 $\mathrm{cm}$, near the iridium anomaly. Interpretation of this succession or turnover, however, is complicated by the factors previously mentioned concerning cherty and porcellanitic sediments with very rare nannofossils, and the possibility of section missing between drilling "biscuits."

\section{Quantitative Results}

Calcareous nannofossil species were counted and placed in one of three categories: Cretaceous, "survivor," and Tertiary. Cretaceous forms are those species considered to have become extinct before or at the end of the Maestrichtian. "Survivor" species are those which were present in the Cretaceous and survived into the Tertiary or had direct descendants surviving into the Tertiary (Perch-Nielsen et al., 1982). Tertiary forms are those that evolved starting at or above the Cretaceous/Tertiary boundary. The results discussed in the following are given in Table 1 and Figure 6. Individual species percent abundances are plotted in Figure 7.

\section{Cretaceous}

The percent abundance of Cretaceous nannofossils ranges between $98 \%$ and $100 \%$ in Samples 121-752B-11R-3, 101-102 cm, to the lowest sample studied, 121-752B-12R-6, 115-116 cm. Because of poor recovery, a sizable gap of approximately $5 \mathrm{~m}$ exists in Core 121-752B-11R between Sample 121-752B-11R-4, $35-36 \mathrm{~cm}$, and Section 121-752B-11R-CC (359.65-364.4 mbsf). Consequently, the graphs of Figures 6 and 7 may not reflect the true character of the Maestrichtian assemblage within this $5-\mathrm{m}$ interval as noted in Figure 7. 
Table 1. Abundance counts of calcareou nannofossils, ODP Samples 121-752B-10R-3, 93-93 cm, through 12-752B-12R-6, 115-116 cm.

\begin{tabular}{|c|c|c|c|c|c|c|c|c|c|c|c|c|c|c|c|c|c|c|c|c|c|}
\hline Age & Zone & $\begin{array}{l}\text { Core, section, } \\
\text { interval }(\mathrm{cm})\end{array}$ & $\begin{array}{l}\text { Depth } \\
\text { (mbsf) }\end{array}$ & 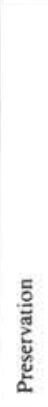 & $\begin{array}{l}\text { हू } \\
\text { हूँ } \\
\text { है }\end{array}$ & 总 & 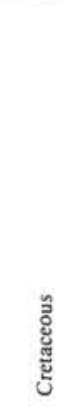 & 竞 & बूँّ & 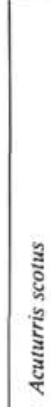 & 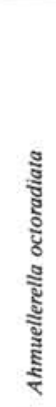 & 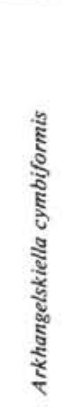 & 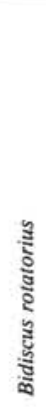 & $\begin{array}{l}\text { हू } \\
\text { हू } \\
\text { है } \\
\text { हू } \\
\text { हूँ }\end{array}$ & 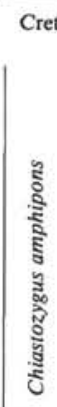 & 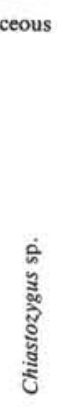 & 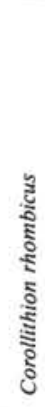 & 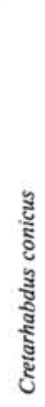 & 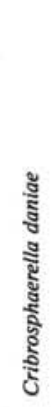 & 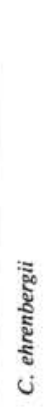 & 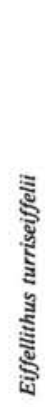 \\
\hline & CP1b & $\begin{array}{l}\text { 10R-3, } 92-93 \\
\text { 10R-3, } 145-146 \\
\text { IOR-4, } 95-96 \\
\text { 10R-4, } 144-145 \\
\text { 10R-5, } 1-2 \\
\text { 10R-5, } 33-34 \\
\text { 10R-5, } 89-90 \\
\text { 10R-5, } 143-144 \\
\text { 10R-6, 32-33 } \\
\text { 10R-6, 86-87 } \\
\text { 10R-6, } 135-136\end{array}$ & $\begin{array}{l}349.02 \\
349.55 \\
350.55 \\
351.04 \\
351.11 \\
351.42 \\
351.99 \\
352.53 \\
352.92 \\
353.46 \\
353.95 \\
\end{array}$ & $\begin{array}{l}\mathrm{M} \\
\mathrm{M} \\
\mathrm{G} \\
\mathrm{M} \\
\mathrm{M} \\
\mathrm{M} \\
\mathrm{M} \\
\mathrm{G} \\
\mathrm{G} \\
\mathrm{M} \\
\mathrm{M}\end{array}$ & $\begin{array}{l}\mathrm{v} \\
\mathrm{V} \\
\mathrm{V} \\
\mathrm{C} \\
\mathrm{C} \\
\mathrm{C} \\
\mathrm{A} \\
\mathrm{A} \\
\mathrm{A} \\
\mathrm{C} \\
\mathrm{C} \\
\mathrm{C}\end{array}$ & $\begin{array}{r}296 \\
301 \\
301 \\
274 \\
267 \\
444 \\
479 \\
427 \\
322 \\
148 \\
8 \\
\end{array}$ & $\begin{array}{r}0 \\
1 \\
0 \\
2 \\
4 \\
3 \\
3 \\
1 \\
1 \\
5 \\
21\end{array}$ & $\begin{array}{r}18 \\
12 \\
9 \\
31 \\
35 \\
58 \\
28 \\
112 \\
195 \\
356 \\
473 \\
\end{array}$ & $\begin{array}{l}314 \\
314 \\
310 \\
307 \\
306 \\
505 \\
510 \\
540 \\
518 \\
504 \\
502 \\
\end{array}$ & $\begin{array}{l}0 \\
0 \\
0 \\
0 \\
1 \\
0 \\
0 \\
0 \\
0 \\
1 \\
1\end{array}$ & $\begin{array}{l}0 \\
0 \\
0 \\
0 \\
0 \\
0 \\
0 \\
0 \\
0 \\
0 \\
0 \\
\end{array}$ & $\begin{array}{l}0 \\
0 \\
0 \\
0 \\
0 \\
0 \\
0 \\
0 \\
0 \\
0 \\
4 \\
\end{array}$ & $\begin{array}{l}0 \\
0 \\
0 \\
0 \\
0 \\
0 \\
0 \\
0 \\
0 \\
0 \\
0\end{array}$ & $\begin{array}{l}0 \\
0 \\
0 \\
0 \\
0 \\
0 \\
0 \\
0 \\
0 \\
0 \\
0 \\
\end{array}$ & $\begin{array}{l}0 \\
0 \\
0 \\
0 \\
0 \\
0 \\
0 \\
0 \\
0 \\
0 \\
0 \\
\end{array}$ & $\begin{array}{l}0 \\
0 \\
0 \\
0 \\
0 \\
0 \\
0 \\
0 \\
0 \\
0 \\
0\end{array}$ & $\begin{array}{l}0 \\
0 \\
0 \\
0 \\
0 \\
0 \\
0 \\
0 \\
0 \\
0 \\
0\end{array}$ & $\begin{array}{l}0 \\
0 \\
0 \\
0 \\
0 \\
0 \\
0 \\
0 \\
0 \\
0 \\
0\end{array}$ & $\begin{array}{l}0 \\
0 \\
0 \\
0 \\
0 \\
0 \\
0 \\
0 \\
0 \\
0 \\
2 \\
\end{array}$ & $\begin{array}{l}0 \\
0 \\
0 \\
0 \\
0 \\
0 \\
0 \\
0 \\
0 \\
0 \\
1 \\
\end{array}$ & $\begin{array}{l}0 \\
0 \\
0 \\
0 \\
0 \\
0 \\
0 \\
0 \\
0 \\
1 \\
0 \\
\end{array}$ \\
\hline ֻั้ & CPla & 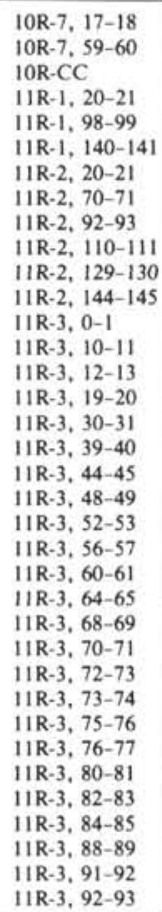 & $\begin{array}{l}354.27 \\
354.69 \\
354.8 \\
355.0 \\
355.78 \\
356.2 \\
356.5 \\
357.0 \\
357.22 \\
357.4 \\
357.59 \\
357.74 \\
357.8 \\
357.9 \\
357.92 \\
357.99 \\
358.1 \\
358.19 \\
358.24 \\
358.28 \\
358.32 \\
355.36 \\
358.4 \\
358.44 \\
358.48 \\
358.5 \\
358.52 \\
358.53 \\
358.55 \\
358.55 \\
358.6 \\
358.61 \\
358.64 \\
358.67 \\
358.71 \\
358.72\end{array}$ & $\begin{array}{l}\mathrm{M} \\
\mathrm{G} \\
\mathrm{G} \\
\mathrm{G} \\
\mathrm{M} \\
\mathrm{M} \\
\mathrm{G} \\
\mathrm{G} \\
\mathrm{M} \\
\mathrm{M} \\
\mathrm{M} \\
\mathrm{M} \\
\mathrm{M} \\
\mathrm{M} \\
\mathrm{M} \\
\mathrm{M} \\
\mathrm{M} \\
\mathrm{G} \\
\mathrm{P} \\
\mathrm{M} \\
\mathrm{M} \\
\mathrm{P} \\
\mathrm{M} \\
\mathrm{M} \\
\mathrm{M} \\
\mathrm{M} \\
\mathrm{P} \\
\mathrm{M} \\
\mathrm{P} \\
\mathrm{P} \\
\mathrm{P} \\
\mathrm{P} \\
\mathrm{P} \\
\mathrm{P} \\
\mathrm{M} \\
\mathrm{M}\end{array}$ & $\begin{array}{l}\text { C } \\
\text { C } \\
\text { C } \\
\text { F } \\
\text { F } \\
\text { F } \\
\text { C } \\
\text { F } \\
\text { C } \\
\text { C } \\
\text { C } \\
\text { C } \\
\text { F } \\
\text { F } \\
\text { F } \\
\text { C } \\
\text { C } \\
\text { C } \\
\text { R } \\
\text { F } \\
\text { F } \\
\text { R } \\
\text { C } \\
\text { C } \\
\text { C } \\
\text { C } \\
\text { C } \\
\text { F } \\
\text { R } \\
\text { R } \\
\text { R } \\
\text { C } \\
\text { R } \\
\text { R } \\
\text { C } \\
\text { C }\end{array}$ & $\begin{array}{l}5 \\
1 \\
1 \\
5 \\
1 \\
0 \\
1 \\
0 \\
1 \\
0 \\
3 \\
5 \\
0 \\
2 \\
0 \\
3 \\
0 \\
2 \\
0 \\
0 \\
0 \\
0 \\
0 \\
0 \\
0 \\
0 \\
x \\
0 \\
0 \\
0 \\
0 \\
0 \\
0 \\
0 \\
0 \\
0\end{array}$ & $\begin{array}{r}13 \\
13 \\
12 \\
29 \\
34 \\
53 \\
30 \\
195 \\
150 \\
309 \\
188 \\
111 \\
134 \\
439 \\
463 \\
60 \\
43 \\
61 \\
4 \\
51 \\
21 \\
16 \\
47 \\
317 \\
278 \\
318 \\
291 \\
286 \\
260 \\
32 \\
1 \\
1 \\
475 \\
41 \\
14 \\
484 \\
486\end{array}$ & $\begin{array}{r}489 \\
485 \\
489 \\
469 \\
470 \\
448 \\
467 \\
309 \\
351 \\
190 \\
311 \\
387 \\
373 \\
111 \\
37 \\
439 \\
460 \\
440 \\
94 \\
453 \\
485 \\
85 \\
456 \\
244 \\
218 \\
203 \\
225 \\
216 \\
240 \\
17 \\
3 \\
39 \\
99 \\
2 \\
31 \\
28\end{array}$ & $\begin{array}{r}507 \\
501 \\
502 \\
503 \\
505 \\
501 \\
498 \\
504 \\
502 \\
499 \\
502 \\
503 \\
507 \\
552 \\
500 \\
502 \\
503 \\
503 \\
98 \\
504 \\
506 \\
101 \\
503 \\
561 \\
496 \\
521 \\
516 \\
502 \\
500 \\
49 \\
4 \\
514 \\
501 \\
161 \\
515 \\
514\end{array}$ & $\begin{array}{l}1 \\
0 \\
0 \\
0 \\
0 \\
2 \\
0 \\
2 \\
1 \\
0 \\
0 \\
0 \\
0 \\
2 \\
1 \\
1 \\
1 \\
1 \\
0 \\
0 \\
0 \\
0 \\
0 \\
0 \\
4 \\
0 \\
1 \\
0 \\
0 \\
0 \\
0 \\
4 \\
0 \\
0 \\
1 \\
1\end{array}$ & $\begin{array}{r}0 \\
0 \\
0 \\
0 \\
0 \\
0 \\
1 \\
1 \\
6 \\
3 \\
10 \\
9 \\
1 \\
9 \\
29 \\
26 \\
2 \\
2 \\
5 \\
0 \\
2 \\
0 \\
0 \\
1 \\
12 \\
13 \\
10 \\
4 \\
8 \\
2 \\
1 \\
0 \\
10 \\
2 \\
0 \\
36 \\
11\end{array}$ & $\begin{array}{r}0 \\
0 \\
1 \\
2 \\
2 \\
9 \\
4 \\
25 \\
25 \\
47 \\
27 \\
23 \\
12 \\
37 \\
59 \\
10 \\
5 \\
7 \\
1 \\
6 \\
7 \\
7 \\
2 \\
10 \\
35 \\
34 \\
51 \\
61 \\
43 \\
46 \\
7 \\
0 \\
76 \\
5 \\
5 \\
31 \\
31 \\
81\end{array}$ & $\begin{array}{l}{ }_{3} \\
0 \\
0 \\
0 \\
0 \\
0 \\
0 \\
0 \\
0 \\
0\end{array}$ & $\begin{array}{l}0 \\
0\end{array}$ & \begin{tabular}{|r}
0 \\
0 \\
0 \\
1 \\
1 \\
1 \\
0 \\
10 \\
2 \\
16 \\
7 \\
6 \\
9 \\
24 \\
37 \\
0 \\
2 \\
2 \\
0 \\
1 \\
0 \\
1 \\
1 \\
20 \\
10 \\
11 \\
8 \\
3 \\
4 \\
0 \\
0 \\
10 \\
1 \\
0 \\
16 \\
12
\end{tabular} & $\begin{array}{r}0 \\
0 \\
0 \\
0 \\
1 \\
0 \\
0 \\
0 \\
1 \\
1 \\
1 \\
5 \\
5 \\
0 \\
1 \\
4 \\
4\end{array}$ & $\begin{array}{l}0 \\
0 \\
0 \\
0 \\
0 \\
0 \\
0 \\
0 \\
0 \\
0 \\
0 \\
0 \\
0 \\
0 \\
0 \\
0 \\
0 \\
1 \\
0 \\
0 \\
0 \\
0 \\
0 \\
0 \\
0 \\
0 \\
0 \\
0 \\
0 \\
0 \\
0 \\
0 \\
0\end{array}$ & $\begin{array}{r}0 \\
0 \\
0 \\
0 \\
1 \\
1 \\
2 \\
4 \\
4 \\
3\end{array}$ & $\begin{array}{r}1 \\
0 \\
0 \\
4 \\
0 \\
7 \\
1 \\
14 \\
6 \\
17 \\
5 \\
5 \\
7 \\
7 \\
40 \\
31 \\
3 \\
2 \\
7 \\
0 \\
5 \\
2 \\
2 \\
0 \\
3 \\
19 \\
21 \\
12 \\
25 \\
21 \\
17 \\
4 \\
0 \\
24 \\
3 \\
3 \\
0 \\
15 \\
13\end{array}$ & $\begin{array}{l}0^{3} \\
1 \\
1 \\
0 \\
1 \\
0\end{array}$ & $\begin{array}{l}1 \\
0 \\
0\end{array}$ \\
\hline 苞 & C. daniae & $\begin{array}{l}11 \mathrm{R}-3,101-102 \\
11 \mathrm{R}-3,110-111 \\
11 \mathrm{R}-3,120-121 \\
1 \mathrm{I}-3,30-131 \\
11 \mathrm{R}-3,3140-141 \\
11 \mathrm{R}-4,10-11 \\
11 \mathrm{R}-4,35-36 \\
11 \mathrm{R}-\mathrm{CC} \\
12 \mathrm{R}-1,40-41 \\
12 \mathrm{R}-1,116-117 \\
12 \mathrm{R}-2,40-41 \\
12 \mathrm{R}-2,115-116 \\
12 \mathrm{R}-3,41-42 \\
12 R-3,115-116 \\
12 \mathrm{R}-4,41-42 \\
12 \mathrm{R}-4,115-116 \\
12 \mathrm{R}-5,41-42 \\
12 \mathrm{R}-5,114-115 \\
12 \mathrm{R}-6,41-42 \\
12 \mathrm{R}-6,115-116\end{array}$ & $\begin{array}{l}358.8 \\
358.9 \\
359.0 \\
359.1 \\
359.2 \\
359.4 \\
359.65 \\
364.4 \\
364.8 \\
365.56 \\
366.3 \\
367.05 \\
367.81 \\
368.55 \\
369.31 \\
370.05 \\
370.81 \\
371.54 \\
372.31 \\
373.05\end{array}$ & $\begin{array}{l}\mathrm{P} \\
\mathrm{P} \\
\mathrm{P} \\
\mathrm{P} \\
\mathrm{P} \\
\mathrm{P} \\
\mathrm{P} \\
\mathrm{P} \\
\mathrm{P} \\
\mathrm{P} \\
\mathrm{P} \\
\mathrm{P} \\
\mathrm{P} \\
\mathrm{P} \\
\mathrm{P} \\
\mathrm{P} \\
\mathrm{P} \\
\mathrm{P} \\
\mathrm{P} \\
\mathrm{P}\end{array}$ & $\begin{array}{l}\text { A } \\
\text { A } \\
\text { A } \\
\text { A } \\
\text { C } \\
\text { C } \\
\text { A } \\
\text { C } \\
\text { A } \\
\text { C } \\
\text { A } \\
\text { A } \\
\text { A } \\
\text { A } \\
\text { A } \\
\text { A } \\
\text { A } \\
\text { A } \\
\text { A } \\
\text { A }\end{array}$ & $\begin{array}{l}0 \\
0 \\
0 \\
0 \\
0 \\
0 \\
0 \\
0 \\
0 \\
0 \\
0 \\
0 \\
0 \\
0 \\
0 \\
0 \\
0 \\
0 \\
0 \\
0\end{array}$ & $\begin{array}{l}495 \\
503 \\
515 \\
509 \\
508 \\
511 \\
504 \\
506 \\
505 \\
505 \\
504 \\
501 \\
499 \\
505 \\
505 \\
502 \\
508 \\
507 \\
509 \\
505\end{array}$ & $\begin{array}{l}7 \\
0 \\
1 \\
0 \\
0 \\
1 \\
0 \\
0 \\
1 \\
1 \\
0 \\
0 \\
1 \\
3 \\
0 \\
0 \\
0 \\
1 \\
0 \\
0\end{array}$ & $\begin{array}{l}502 \\
503 \\
516 \\
509 \\
508 \\
512 \\
504 \\
506 \\
506 \\
506 \\
504 \\
501 \\
500 \\
508 \\
505 \\
502 \\
508 \\
508 \\
509 \\
505\end{array}$ & $\begin{array}{r}7 \\
1 \\
3 \\
1 \\
1 \\
3 \\
3 \\
0 \\
3 \\
4 \\
4 \\
10 \\
3 \\
16 \\
3 \\
0 \\
0 \\
0 \\
0 \\
12\end{array}$ & $\begin{array}{r}9 \\
9 \\
14 \\
13 \\
6 \\
6 \\
4 \\
1 \\
3 \\
0 \\
2 \\
4 \\
1 \\
3 \\
2 \\
0 \\
0 \\
0 \\
5 \\
0\end{array}$ & $\begin{array}{r}74 \\
78 \\
85 \\
75 \\
63 \\
46 \\
56 \\
43 \\
46 \\
62 \\
39 \\
94 \\
41 \\
131 \\
98 \\
47 \\
48 \\
41 \\
42 \\
111\end{array}$ & $\begin{array}{l}0 \\
0 \\
0 \\
0 \\
0 \\
0 \\
0 \\
0 \\
0 \\
0 \\
0 \\
0 \\
0 \\
0 \\
0 \\
0 \\
0 \\
0 \\
0 \\
0\end{array}$ & $\begin{array}{l}0 \\
0 \\
0 \\
0 \\
2 \\
9 \\
1\end{array}$ & $\begin{array}{r}17 \\
13 \\
20 \\
5 \\
14 \\
4 \\
9 \\
1 \\
13 \\
3 \\
14 \\
4 \\
1 \\
2 \\
5 \\
0 \\
10 \\
7 \\
15 \\
7\end{array}$ & $\begin{array}{l} \\
0 \\
0 \\
0 \\
0 \\
0 \\
0 \\
0 \\
7 \\
1 \\
6 \\
1 \\
0 \\
0 \\
0 \\
0 \\
0 \\
0 \\
0 \\
0\end{array}$ & $\begin{array}{l}0 \\
0 \\
0 \\
0 \\
0 \\
0 \\
0 \\
0 \\
0 \\
0 \\
0 \\
0 \\
0 \\
0 \\
0 \\
0 \\
0 \\
0 \\
0 \\
0\end{array}$ & \begin{tabular}{r|}
8 \\
8 \\
10 \\
9 \\
3 \\
5 \\
5 \\
10 \\
3 \\
7 \\
1 \\
7 \\
3 \\
4 \\
4 \\
1 \\
5 \\
7 \\
15 \\
6 \\
3 \\
3 \\
8
\end{tabular} & $\begin{array}{l}11 \\
12 \\
10 \\
19 \\
13 \\
25 \\
16 \\
15 \\
43 \\
40 \\
26 \\
34 \\
37 \\
29 \\
30 \\
28 \\
26 \\
19 \\
22 \\
20\end{array}$ & $\begin{array}{l}0 \\
0 \\
1 \\
2 \\
2 \\
1 \\
4 \\
1 \\
4 \\
2 \\
2 \\
3 \\
3 \\
8 \\
5 \\
5 \\
1 \\
0 \\
3 \\
2 \\
4\end{array}$ & $\begin{array}{r}4 \\
3 \\
1 \\
3 \\
7 \\
4 \\
6 \\
1 \\
4 \\
4 \\
2 \\
5 \\
8 \\
12 \\
8 \\
3 \\
15 \\
5 \\
10 \\
5\end{array}$ \\
\hline
\end{tabular}

${ }^{a}$ Reworked lower Maestrichtian species.
$\mathrm{X}=$ present but not within 500 count. 


\begin{tabular}{|c|c|c|c|c|c|c|c|c|c|c|c|c|c|c|c|c|c|c|c|c|c|c|c|}
\hline 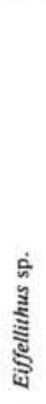 & 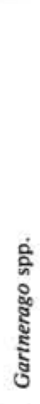 & 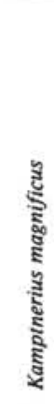 & 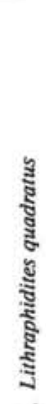 & 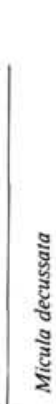 & 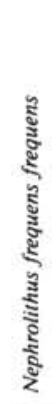 & 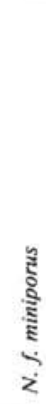 & 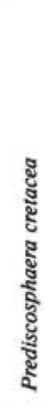 & 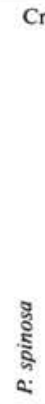 & $\begin{array}{l}\mathrm{J} \\
\text { है } \\
\text { Q }\end{array}$ & 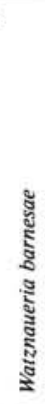 & 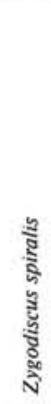 & 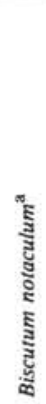 & 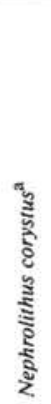 & 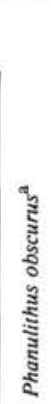 & 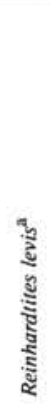 & 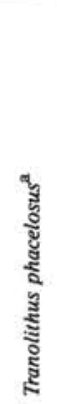 & 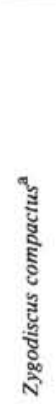 & 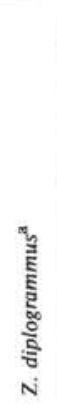 & 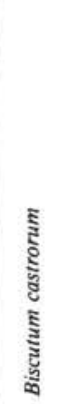 & 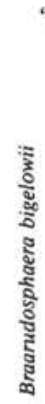 & 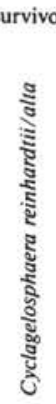 & 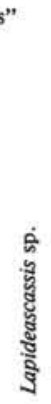 & 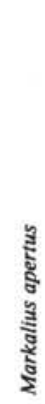 \\
\hline 0 & 0 & 0 & 0 & 0 & 0 & 0 & 0 & 0 & 0 & 0 & 0 & 0 & 0 & 0 & 0 & 0 & 0 & 0 & 0 & 0 & 0 & 0 & 0 \\
\hline 0 & 0 & 0 & 0 & 1 & 0 & 0 & 0 & 0 & 0 & 0 & 0 & 0 & 0 & 0 & 0 & 0 & 0 & 0 & 1 & 0 & 0 & 0 & 0 \\
\hline 0 & 0 & 0 & 0 & 0 & 0 & 0 & 0 & 0 & 0 & 0 & 0 & 0 & 0 & 0 & 0 & 0 & 0 & 0 & 0 & 0 & 0 & 0 & 0 \\
\hline 0 & 0 & 0 & 0 & 0 & 0 & 0 & 0 & 0 & 0 & 0 & 0 & 0 & 0 & 0 & 0 & 0 & 0 & 0 & 2 & 0 & 0 & 0 & 0 \\
\hline 0 & 0 & 2 & 0 & 0 & 0 & 0 & 0 & 0 & 0 & 0 & 0 & 0 & 1 & 0 & 0 & 0 & 0 & 0 & 2 & 0 & 0 & 0 & 0 \\
\hline 0 & 0 & 0 & 0 & 3 & 0 & 0 & 0 & 0 & 0 & 0 & 0 & 0 & 0 & 0 & 0 & 0 & 0 & 0 & 9 & 0 & 0 & 0 & 0 \\
\hline 0 & 1 & 0 & 0 & 2 & 0 & 0 & 0 & 0 & 0 & 0 & 0 & 0 & 0 & 0 & 0 & 0 & 0 & 0 & 4 & 0 & 0 & 0 & 0 \\
\hline 0 & 0 & 0 & 0 & 0 & 0 & 0 & 0 & 0 & 0 & 1 & 0 & 0 & 0 & 0 & 0 & 0 & 0 & 0 & 23 & 0 & 0 & 0 & 0 \\
\hline 0 & 0 & 0 & 0 & 0 & 0 & 0 & 0 & 0 & 0 & 0 & 0 & 0 & 0 & 0 & 1 & 0 & 0 & 0 & 24 & 0 & 1 & 0 & 5 \\
\hline 0 & 0 & 1 & 0 & 1 & 0 & 0 & 0 & 0 & 0 & 0 & 0 & 0 & 0 & 0 & 1 & 0 & 0 & 0 & 44 & 0 & 0 & 0 & 1 \\
\hline 0 & 1 & 2 & 0 & 5 & 0 & 0 & 0 & 0 & 4 & 1 & 0 & 0 & 0 & 0 & 0 & 0 & 0 & 0 & 38 & 0 & 0 & 0 & 1 \\
\hline 0 & 0 & 3 & 0 & 2 & 0 & 0 & 0 & 1 & 0 & 0 & $\overline{0}$ & 0 & 1 & 0 & 3 & 0 & 0 & 0 & 18 & 0 & 1 & 0 & 0 \\
\hline 0 & 1 & 0 & 0 & 4 & 1 & 0 & 2 & 2 & 1 & 0 & 0 & 0 & 0 & 0 & 1 & 0 & 0 & 0 & 60 & 0 & 0 & 0 & 2 \\
\hline 0 & 0 & 2 & 0 & 5 & 1 & 0 & 1 & 0 & 0 & 1 & 0 & 0 & 2 & 0 & 0 & 0 & 0 & 0 & 21 & 0 & 1 & 0 & 1 \\
\hline 0 & 0 & 3 & 0 & 2 & 3 & 0 & I & 0 & 5 & 2 & 0 & 0 & 0 & 0 & 1 & 0 & 0 & 0 & 31 & 0 & 0 & 0 & 3 \\
\hline 0 & 0 & 3 & 0 & 11 & 4 & 0 & 3 & 0 & 2 & 1 & 3 & 0 & 0 & 0 & 1 & 0 & 0 & 0 & 29 & 0 & 0 & 0 & 3 \\
\hline 2 & 0 & 7 & 0 & 10 & 3 & 1 & 1 & 0 & 1 & 4 & 1 & 0 & 0 & 0 & 0 & 0 & 0 & 0 & 30 & 0 & 3 & 0 & 2 \\
\hline 0 & 0 & 2 & 0 & 5 & 4 & 1 & 1 & 0 & 4 & 2 & 0 & 0 & 0 & 0 & 1 & 0 & 1 & 0 & 25 & 0 & 2 & 2 & 5 \\
\hline 0 & 1 & 18 & 0 & 37 & 24 & 7 & 6 & 1 & 24 & 7 & 4 & 0 & 0 & 0 & $i$ & 2 & 0 & 0 & 29 & i & 14 & 0 & i \\
\hline 2 & 0 & 5 & 0 & 37 & 27 & 2 & 7 & 2 & 14 & 0 & 4 & 0 & 0 & 0 & 0 & 0 & 0 & 0 & 24 & 0 & 2 & 0 & 0 \\
\hline 3 & 1 & 32 & 0 & 56 & 48 & 10 & 3 & 0 & 36 & 3 & 9 & 0 & 0 & 0 & 1 & 0 & 0 & 0 & 9 & 0 & 0 & 0 & 0 \\
\hline 2 & 2 & 21 & 0 & 31 & 44 & 4 & 11 & 0 & 15 & 1 & 3 & 0 & 0 & 0 & 0 & 0 & 0 & 0 & 30 & 0 & 2 & 1 & 1 \\
\hline 0 & 0 & 9 & 0 & 18 & 18 & i & i & 1 & 12 & 3 & 4 & 0 & 0 & 0 & 1 & 0 & 0 & 0 & 25 & 0 & 2 & 0 & 0 \\
\hline 0 & 0 & 5 & 0 & 15 & 23 & 2 & 6 & 0 & 35 & 0 & 0 & 0 & 0 & 0 & I & 1 & 1 & 0 & 9 & 0 & 0 & 0 & 1 \\
\hline 8 & 9 & 25 & 0 & 37 & 76 & 19 & 16 & 0 & 55 & 6 & 13 & 0 & 0 & 0 & 1 & 0 & 2 & 0 & 4 & 0 & 2 & 0 & 0 \\
\hline 3 & 2 & 35 & 0 & 71 & 76 & 20 & 13 & 0 & 47 & 4 & 19 & 0 & 0 & 0 & 1 & 0 & 0 & 1 & 1 & 0 & 1 & 0 & 0 \\
\hline 0 & 2 & 2 & 0 & 5 & 13 & 2 & 3 & 0 & 14 & 0 & 3 & 0 & 0 & 0 & 0 & 0 & 0 & 0 & 27 & 0 & 3 & 0 & 0 \\
\hline 0 & 0 & 4 & 0 & 5 & 10 & 1 & 1 & 0 & 7 & 0 & 0 & 0 & 0 & 0 & 0 & 0 & 0 & 0 & 44 & 0 & 0 & 0 & 2 \\
\hline 0 & 0 & 4 & 0 & 4 & 13 & 0 & 2 & 0 & 7 & 1 & 0 & 0 & 0 & 0 & 0 & 1 & 0 & 1 & 90 & 0 & 0 & 0 & 0 \\
\hline 0 & 0 & 0 & 0 & 0 & 1 & 1 & 0 & 0 & 0 & 0 & 0 & 0 & 0 & 0 & 0 & 0 & 0 & 0 & 20 & 0 & 0 & 0 & 0 \\
\hline 0 & 0 & 4 & 0 & 6 & 8 & 0 & 5 & 0 & 12 & 0 & 0 & 0 & 0 & 0 & 0 & 0 & 0 & 0 & 47 & 0 & I & 1 & 0 \\
\hline 0 & 0 & 2 & 0 & 0 & 4 & 1 & 3 & 0 & 2 & 0 & 0 & 0 & 0 & 0 & 0 & 0 & 0 & 0 & 29 & 1 & 1 & 0 & 0 \\
\hline 0 & 0 & 2 & 0 & 4 & 3 & 0 & 2 & 0 & 0 & 1 & 0 & 0 & 0 & 0 & 0 & 0 & 1 & 0 & 12 & 1 & 0 & 0 & 0 \\
\hline 0 & 1 & 2 & 0 & 3 & 12 & 0 & 3 & 0 & 6 & 0 & 1 & 0 & 0 & 0 & 0 & 0 & 0 & 0 & 91 & 0 & 4 & 0 & 0 \\
\hline 0 & 2 & 39 & 0 & 39 & 71 & 7 & 7 & 2 & 42 & 4 & 4 & 0 & 0 & 0 & 0 & 0 & 0 & 0 & 38 & 3 & 4 & 0 & 0 \\
\hline 0 & 1 & 32 & 0 & 14 & 66 & 7 & 8 & 0 & 46 & 0 & 8 & 0 & 0 & 0 & 0 & 0 & 0 & 0 & 37 & 0 & 5 & 0 & 0 \\
\hline 0 & 2 & 21 & 0 & 22 & 90 & 4 & 9 & 0 & 63 & 3 & 6 & 0 & 0 & 0 & 1 & 0 & 0 & 0 & 31 & 0 & 6 & 0 & 0 \\
\hline 0 & 0 & 30 & 0 & 72 & 39 & 5 & 15 & 0 & 19 & 0 & 8 & 0 & 0 & 0 & 0 & 0 & i & 0 & 65 & 0 & 6 & 0 & 0 \\
\hline 1 & 1 & 14 & 0 & 23 & 71 & 6 & 6 & 0 & 61 & 1 & 10 & 0 & 0 & 0 & 0 & 0 & 0 & 0 & 25 & 0 & 3 & 0 & 0 \\
\hline 0 & 3 & 31 & 0 & 77 & 45 & 2 & 12 & 0 & 11 & 0 & 2 & 0 & 0 & 0 & 0 & 0 & 0 & 0 & 3 & 0 & 3 & 0 & 0 \\
\hline 0 & 0 & 2 & 0 & 5 & 11 & 0 & 1 & 0 & 1 & 0 & 0 & 0 & 0 & 0 & 0 & 0 & 0 & 0 & 0 & 0 & 0 & 0 & 0 \\
\hline 0 & 0 & 0 & 0 & 0 & 0 & 0 & 0 & 0 & 0 & 0 & 0 & 0 & 0 & 0 & 0 & 0 & 0 & 0 & 0 & 0 & 0 & 0 & 0 \\
\hline 2 & 1 & 59 & 0 & 93 & 70 & 5 & 27 & 0 & 54 & 4 & 15 & 0 & 0 & 0 & 0 & 0 & 0 & 0 & 11 & 0 & 0 & 0 & 0 \\
\hline 0 & 0 & 5 & 0 & 13 & 7 & 0 & 2 & 0 & 2 & 0 & 0 & 0 & 0 & 0 & 0 & 0 & 0 & 0 & 3 & 0 & 0 & 0 & 0 \\
\hline 0 & 0 & 1 & 0 & 5 & 1 & 0 & 0 & 0 & 1 & 0 & 0 & 0 & 0 & 0 & 0 & 0 & 0 & 0 & 0 & 0 & 0 & 0 & 0 \\
\hline 6 & 3 & 39 & 0 & 37 & 83 & 14 & 12 & 0 & 152 & 2 & 10 & 0 & 0 & 0 & 0 & 0 & 0 & 0 & 10 & 0 & 0 & 0 & 0 \\
\hline 0 & 2 & 26 & 0 & 143 & 71 & 1 & 25 & 0 & 55 & 5 & 19 & 0 & 0 & 0 & 0 & 0 & 0 & 0 & 11 & 0 & 0 & 0 & 0 \\
\hline 0 & 3 & 41 & 0 & 149 & 90 & 3 & 19 & 0 & 40 & 4 & 15 & 0 & 0 & 0 & 0 & 0 & 0 & 0 & 4 & 0 & 0 & 0 & 0 \\
\hline 0 & 2 & 42 & 0 & 1 & 92 & 4 & i & 0 & & 7 & & & & 0 & 0 & 0 & 0 & 0 & 0 & 0 & 0 & 0 & 0 \\
\hline 0 & 0 & 41 & 0 & 189 & 82 & 4 & 2 & 0 & 1 & 3 & 10 & 0 & 0 & 0 & 0 & 0 & 0 & 0 & 0 & 0 & 0 & 1 & 0 \\
\hline 1 & 3 & 45 & 0 & 184 & 75 & 6 & 15 & 0 & 33 & 4 & 16 & 0 & 0 & 0 & 0 & 0 & 0 & 0 & 0 & 0 & 0 & 0 & 0 \\
\hline 1 & 1 & 51 & 0 & 195 & 95 & 2 & 14 & 0 & 24 & 2 & 14 & 0 & 0 & 0 & 0 & 0 & 0 & 0 & 0 & 0 & 0 & 0 & 0 \\
\hline i & 0 & 47 & 0 & 273 & 43 & 6 & 18 & 0 & 18 & 3 & 8 & 0 & 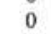 & 0 & 0 & 0 & 0 & 0 & 0 & 0 & 0 & 0 & 0 \\
\hline 0 & 0 & 45 & 0 & 223 & 42 & 7 & 16 & 0 & 48 & 4 & 11 & 0 & 0 & 0 & 0 & 0 & 0 & 0 & 0 & 0 & 0 & 0 & 0 \\
\hline 0 & 0 & 46 & 0 & 360 & 18 & 1 & 11 & 0 & 1 & 4 & 0 & 0 & 0 & 0 & 0 & 0 & 0 & 0 & 0 & 0 & 0 & 0 & 0 \\
\hline 5 & 3 & 77 & 0 & 33 & 82 & 23 & 10 & 0 & 94 & 1 & 47 & 0 & 0 & 0 & 0 & 0 & 0 & 0 & 0 & 0 & 0 & 0 & 0 \\
\hline 1 & 2 & 92 & 0 & 113 & 54 & 12 & 31 & 1 & 34 & 1 & 47 & 0 & 0 & 0 & 0 & 0 & 0 & 0 & 0 & 0 & 0 & 0 & 0 \\
\hline 1 & 5 & 91 & 0 & 82 & 5 & 16 & 3 & 0 & 7 & 0 & 38 & 0 & 0 & 0 & 0 & 0 & 1 & 0 & 0 & 0 & 0 & 0 & 0 \\
\hline 2 & 5 & 108 & 0 & 44 & 44 & 10 & 2 & 1 & 70 & 0 & 21 & 0 & 0 & 0 & 0 & 0 & 0 & 0 & 0 & 0 & 0 & 0 & 0 \\
\hline 2 & 3 & 129 & $x$ & 66 & 46 & 12 & 48 & 0 & 77 & 2 & 7 & 1 & 0 & 0 & 2 & 0 & 0 & 0 & 0 & 0 & 0 & 0 & 0 \\
\hline 1 & 2 & 141 & 0 & 81 & 23 & 6 & 37 & 0 & 10 & 3 & 1 & 0 & 0 & 1 & 0 & 0 & 0 & 0 & 0 & 0 & 0 & 0 & 0 \\
\hline 3 & 4 & 76 & 0 & 98 & 72 & 14 & 2 & 0 & 37 & 0 & 15 & 0 & 0 & 0 & 3 & 0 & 0 & 0 & 0 & 0 & 0 & 0 & 0 \\
\hline 0 & 0 & 101 & 0 & 237 & 33 & 12 & 22 & 0 & 9 & 1 & 0 & 0 & 0 & 0 & 1 & 0 & 0 & 0 & 0 & 0 & 0 & 0 & 0 \\
\hline 0 & 4 & 112 & 0 & 106 & 52 & so & 21 & 2 & 32 & 0 & 15 & 0 & 0 & 0 & 0 & 0 & 0 & 0 & 0 & 0 & 0 & 0 & 0 \\
\hline 0 & 0 & 77 & 0 & 220 & 44 & 23 & 14 & 0 & 42 & 0 & 6 & 0 & 0 & 0 & 0 & 0 & 0 & 0 & 0 & 0 & 0 & 0 & 0 \\
\hline 0 & 3 & 63 & 0 & 94 & 94 & 45 & 27 & 1 & 70 & 3 & 10 & 0 & 0 & 0 & 0 & 0 & 0 & 0 & 0 & 0 & 0 & 0 & 0 \\
\hline 1 & 1 & 134 & 0 & 89 & 29 & 12 & 34 & i & 10 & 2 & 24 & 0 & 0 & 0 & 1 & 0 & 0 & 0 & 0 & 0 & 0 & 0 & 0 \\
\hline
\end{tabular}


Table 1 (continued).

\begin{tabular}{|c|c|c|c|c|c|c|c|c|c|c|c|c|c|c|c|c|c|c|}
\hline \multirow[t]{2}{*}{ Age } & Zone & $\begin{array}{l}\text { Core, section, } \\
\text { interval (cm) }\end{array}$ & $\begin{array}{l}\text { Depth } \\
\text { (mbsf) }\end{array}$ & $\begin{array}{l}\text { है } \\
\text { है } \\
\text { ¿ }\end{array}$ & 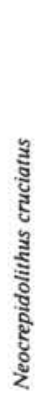 & 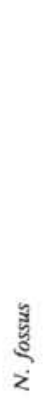 & $\begin{array}{l}\text { w } \\
\text { हूँ } \\
\text { ஜ } \\
z\end{array}$ & 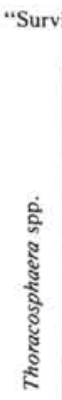 & 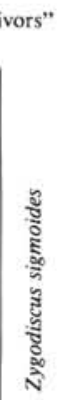 & 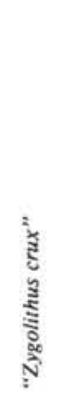 & 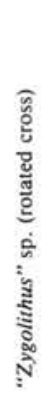 & 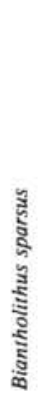 & 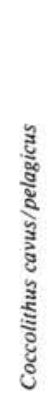 & 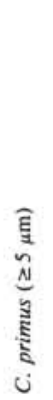 & 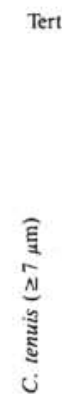 & 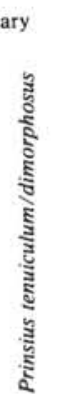 & 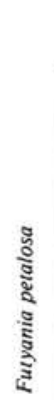 & 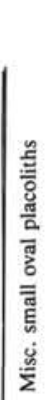 \\
\hline & CPIb & $\begin{array}{l}\text { 10R-3, 92-93 } \\
\text { 10R-3, 145-146 } \\
\text { 10R-4, 95-96 } \\
\text { 10R-4, 144-145 } \\
\text { 10R-5, 1-2 } \\
\text { 10R-5, 33-34 } \\
\text { 10R-5, 89-90 } \\
\text { 10R-5, 143-144 } \\
\text { 10R-6, 32-33 } \\
\text { 10R-6, 86-87 } \\
\text { 10R-6, 135-136 }\end{array}$ & \begin{tabular}{l|}
349.02 \\
349.55 \\
350.55 \\
351.04 \\
351.11 \\
351.42 \\
351.99 \\
352.53 \\
352.92 \\
353.46 \\
353.95 \\
\end{tabular} & $\begin{array}{r}1 \\
1 \\
0 \\
0 \\
0 \\
0 \\
0 \\
6 \\
10 \\
28 \\
42 \\
\end{array}$ & $\begin{array}{r}0 \\
0 \\
0 \\
0 \\
0 \\
2 \\
1 \\
20 \\
31 \\
17 \\
35 \\
\end{array}$ & $\begin{array}{l}1 \\
0 \\
0 \\
0 \\
0 \\
0 \\
0 \\
1 \\
8 \\
0 \\
0 \\
\end{array}$ & $\begin{array}{l}0 \\
0 \\
0 \\
0 \\
0 \\
0 \\
0 \\
0 \\
1 \\
0 \\
0\end{array}$ & $\begin{array}{r}2 \\
0 \\
1 \\
2 \\
2 \\
1 \\
1 \\
5 \\
8 \\
26 \\
40 \\
\end{array}$ & $\begin{array}{r}14 \\
10 \\
8 \\
27 \\
31 \\
46 \\
22 \\
57 \\
107 \\
240 \\
317 \\
\end{array}$ & $\begin{array}{l}0 \\
0 \\
0 \\
0 \\
0 \\
0 \\
0 \\
0 \\
0 \\
0 \\
0 \\
0\end{array}$ & $\begin{array}{l}0 \\
0 \\
0 \\
0 \\
0 \\
0 \\
0 \\
0 \\
1 \\
0 \\
0\end{array}$ & $\begin{array}{l}0 \\
0 \\
0 \\
0 \\
0 \\
0 \\
0 \\
0 \\
0 \\
0 \\
0\end{array}$ & $\begin{array}{r}49 \\
35 \\
2 \\
27 \\
19 \\
44 \\
20 \\
0 \\
1 \\
0 \\
0\end{array}$ & $\begin{array}{r}12 \\
3 \\
0 \\
0 \\
2 \\
12 \\
26 \\
19 \\
25 \\
0 \\
1 \\
\end{array}$ & $\begin{array}{r}52 \\
57 \\
18 \\
126 \\
148 \\
178 \\
112 \\
200 \\
177 \\
140 \\
3 \\
\end{array}$ & $\begin{array}{r}182 \\
205 \\
282 \\
121 \\
97 \\
207 \\
314 \\
143 \\
91 \\
4 \\
0 \\
\end{array}$ & $\begin{array}{l}0 \\
0 \\
0 \\
0 \\
0 \\
0 \\
0 \\
1 \\
0 \\
0 \\
0\end{array}$ & $\begin{array}{r}0 \\
0 \\
0 \\
0 \\
0 \\
1 \\
1 \\
10 \\
4 \\
2 \\
1 \\
\end{array}$ \\
\hline 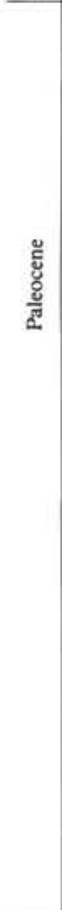 & CPla & $\begin{array}{l}10 R-7,17-18 \\
10 R-7,59-60 \\
10 R-C C \\
11 R-1,20-21 \\
11 R-1,98-99 \\
11 R-1,140-141 \\
11 R-2,20-21 \\
11 R-2,70-71 \\
11 R-2,92-93 \\
11 R-2,110-111 \\
11 R-2,129-130 \\
11 R-2,144-145 \\
11 R-3,0-1 \\
11 R-3,10-11 \\
11 R-3,12-13 \\
11 R-3,19-20 \\
11 R-3,30-31 \\
11 R-3,39-40 \\
11 R-3,44-45 \\
11 R-3,48-49 \\
11 R-3,52-53 \\
11 R-3,56-57 \\
11 R-3,60-61 \\
11 R-3,64-65 \\
11 R-3,68-69 \\
11 R-3,70-71 \\
11 R-3,72-73 \\
11 R-3,73-74 \\
11 R-3,75-76 \\
11 R-3,76-77 \\
11 R-3,80-81 \\
11 R-3,82-83 \\
11 R-3,84-85 \\
11 R-3,88-89 \\
11 R-3,91-92 \\
11 R-3,92-93\end{array}$ & $\begin{array}{l}354.27 \\
354.69 \\
354.8 \\
355.0 \\
355.78 \\
356.2 \\
356.5 \\
357.0 \\
357.22 \\
357.4 \\
357.59 \\
357.74 \\
357.8 \\
357.9 \\
357.92 \\
357.99 \\
358.1 \\
358.19 \\
358.24 \\
358.28 \\
358.32 \\
358.36 \\
358.4 \\
358.44 \\
358.48 \\
358.5 \\
358.52 \\
358.53 \\
358.55 \\
358.55 \\
358.6 \\
358.61 \\
358.64 \\
358.67 \\
358.71 \\
358.72\end{array}$ & $\begin{aligned} 27 \\
53 \\
11 \\
55 \\
59 \\
46 \\
41 \\
19 \\
14 \\
8 \\
8 \\
14 \\
36 \\
2 \\
5 \\
4 \\
31 \\
48 \\
55 \\
4 \\
20 \\
14 \\
5 \\
27 \\
22 \\
12 \\
16 \\
40 \\
10 \\
14 \\
0 \\
0 \\
3 \\
0 \\
0 \\
1 \\
3\end{aligned}$ & $\begin{array}{r}7 \\
20 \\
26 \\
32 \\
51 \\
23 \\
31 \\
31 \\
15 \\
38 \\
15 \\
3 \\
2 \\
3 \\
3 \\
7 \\
9 \\
90 \\
0 \\
0 \\
4 \\
12 \\
4 \\
5 \\
6 \\
4 \\
0 \\
2 \\
0 \\
0 \\
0 \\
0 \\
0 \\
0 \\
0 \\
0 \\
0\end{array}$ & $\begin{array}{l}0 \\
0 \\
0 \\
0 \\
0 \\
0 \\
0 \\
0 \\
0 \\
0 \\
0 \\
0 \\
0 \\
0 \\
0 \\
0 \\
0 \\
0 \\
0 \\
0 \\
0 \\
0 \\
0 \\
0 \\
0 \\
0 \\
0 \\
0 \\
0 \\
0 \\
0 \\
0 \\
0 \\
0 \\
0 \\
0\end{array}$ & $\begin{array}{r}0 \\
1 \\
0 \\
5 \\
15 \\
1 \\
2 \\
20 \\
0 \\
5 \\
2 \\
0 \\
1 \\
0 \\
0 \\
2 \\
0 \\
0 \\
1 \\
0 \\
0 \\
0 \\
2 \\
2 \\
0 \\
0 \\
1 \\
1 \\
0 \\
0 \\
0 \\
0 \\
0 \\
0 \\
0 \\
0\end{array}$ & $\begin{array}{r}41 \\
62 \\
49 \\
72 \\
136 \\
70 \\
60 \\
11 \\
42 \\
32 \\
79 \\
18 \\
103 \\
15 \\
2 \\
82 \\
96 \\
15 \\
8 \\
40 \\
59 \\
6 \\
34 \\
20 \\
35 \\
15 \\
22 \\
38 \\
46 \\
8 \\
2 \\
4 \\
1 \\
0 \\
9 \\
8\end{array}$ & $\begin{array}{r}395 \\
287 \\
380 \\
262 \\
171 \\
264 \\
299 \\
182 \\
251 \\
96 \\
166 \\
302 \\
250 \\
70 \\
23 \\
260 \\
258 \\
214 \\
1 \\
340 \\
369 \\
57 \\
293 \\
149 \\
125 \\
135 \\
89 \\
139 \\
174 \\
9 \\
1 \\
1 \\
21 \\
5 \\
2 \\
11 \\
6\end{array}$ & $\begin{array}{r}1 \\
1 \\
0 \\
0 \\
7 \\
5 \\
7 \\
1 \\
1 \\
3 \\
2 \\
1 \\
0 \\
3 \\
11 \\
3 \\
27 \\
3 \\
3 \\
0 \\
1 \\
0 \\
0 \\
0 \\
0 \\
0 \\
0 \\
0 \\
0 \\
0 \\
0 \\
0 \\
0\end{array}$ & 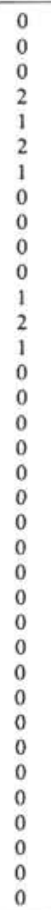 & 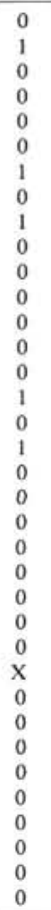 & $\begin{array}{l}0 \\
0 \\
0 \\
0 \\
9 \\
0 \\
0 \\
0 \\
0 \\
0 \\
0 \\
0 \\
0 \\
0 \\
0 \\
0 \\
0 \\
0 \\
0 \\
0 \\
0 \\
0 \\
0 \\
0 \\
0 \\
0 \\
0 \\
0 \\
0 \\
0 \\
0 \\
0 \\
0 \\
0 \\
0 \\
0 \\
0 \\
0 \\
0 \\
0 \\
0\end{array}$ & $\begin{array}{l}3 \\
0 \\
0 \\
0 \\
0 \\
0 \\
0 \\
0 \\
0 \\
0 \\
0 \\
0 \\
0 \\
0 \\
0 \\
0 \\
0 \\
0 \\
0 \\
0 \\
0 \\
0 \\
0 \\
0 \\
0 \\
0 \\
0 \\
0 \\
0 \\
0 \\
0 \\
0 \\
0 \\
0 \\
0 \\
0 \\
0 \\
0 \\
0 \\
0 \\
0 \\
0 \\
0\end{array}$ & $\begin{array}{l}0 \\
0 \\
0 \\
0 \\
0 \\
0 \\
0 \\
0 \\
0 \\
0 \\
0 \\
0 \\
0 \\
0 \\
0 \\
0 \\
0 \\
0 \\
0 \\
0 \\
0 \\
0 \\
0 \\
0 \\
0 \\
0 \\
0 \\
0 \\
0 \\
0 \\
0 \\
0 \\
0 \\
0 \\
0 \\
0 \\
0 \\
0 \\
0 \\
0 \\
0 \\
0 \\
0 \\
0 \\
0 \\
0\end{array}$ & 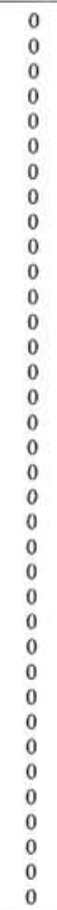 & $\begin{array}{l}0 \\
0 \\
0 \\
0 \\
0 \\
0 \\
0 \\
0 \\
0 \\
0 \\
0 \\
0 \\
0 \\
0 \\
0 \\
0 \\
0 \\
0 \\
0 \\
0 \\
0 \\
0\end{array}$ & $\begin{array}{l}1 \\
1 \\
0 \\
1 \\
5 \\
5 \\
0 \\
0 \\
0 \\
0 \\
0 \\
0 \\
0 \\
3 \\
5 \\
5 \\
0 \\
2 \\
0 \\
2 \\
2 \\
0 \\
1 \\
0 \\
0 \\
0 \\
0 \\
0\end{array}$ \\
\hline 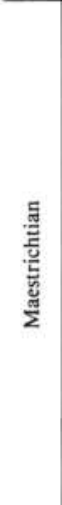 & C. daniae & $\begin{array}{l}11 R-3,101-102 \\
11 R-3,110-111 \\
11 R-3,120-121 \\
11 R-3,130-131 \\
11 R-3,140-141 \\
11 R-4,10-11 \\
11 R-4,35-36 \\
11 R-C C \\
12 R-1,40-41 \\
12 R-1,116-117 \\
12 R-2,40-41 \\
12 R-2,115-116 \\
12 R-3,41-42 \\
12 R-3,115-116 \\
12 R-4,41-42 \\
12 R-4,115-116 \\
12 R-5,41-42 \\
12 R-5,114-115 \\
12 R-6,41-42 \\
12 R-6,115-116\end{array}$ & $\begin{array}{l}358.8 \\
358.9 \\
359.0 \\
359.1 \\
359.2 \\
359.4 \\
359.65 \\
364.4 \\
364.8 \\
365.56 \\
366.3 \\
367.05 \\
367.81 \\
368.55 \\
369.31 \\
370.05 \\
370.81 \\
371.54 \\
372.31 \\
373.05\end{array}$ & $\begin{array}{l}0 \\
0 \\
0 \\
0 \\
0 \\
0 \\
0 \\
0 \\
1 \\
1 \\
0 \\
0 \\
1 \\
3 \\
0 \\
0 \\
0 \\
0 \\
0 \\
0\end{array}$ & $\begin{array}{l}0 \\
0 \\
0 \\
0 \\
0 \\
0 \\
0 \\
0 \\
0 \\
0 \\
0 \\
0 \\
0 \\
0 \\
0 \\
0 \\
0 \\
0 \\
0 \\
0\end{array}$ & $\begin{array}{l}0 \\
0 \\
0 \\
0 \\
0 \\
0 \\
0 \\
0 \\
0 \\
0 \\
0 \\
0 \\
0 \\
0 \\
0 \\
0 \\
0 \\
0 \\
0 \\
0\end{array}$ & $\begin{array}{l}0 \\
0 \\
0 \\
0 \\
0 \\
1 \\
0 \\
0 \\
0 \\
0 \\
0 \\
0 \\
0 \\
0 \\
0 \\
0 \\
0 \\
0 \\
0 \\
0\end{array}$ & $\begin{array}{l}3 \\
0 \\
0 \\
0 \\
0 \\
0 \\
0 \\
0 \\
0 \\
0 \\
0 \\
0 \\
0 \\
0 \\
0 \\
0 \\
0 \\
0 \\
0 \\
0\end{array}$ & $\begin{array}{l}0 \\
0 \\
0 \\
0 \\
0 \\
0 \\
0 \\
0 \\
0 \\
0 \\
0 \\
0 \\
0 \\
0 \\
0 \\
0 \\
0 \\
0 \\
1 \\
0 \\
0\end{array}$ & $\begin{array}{l}0 \\
0 \\
0 \\
0 \\
0 \\
0 \\
0 \\
0 \\
0 \\
0 \\
0 \\
0 \\
0 \\
0 \\
0 \\
0 \\
0 \\
0 \\
0 \\
0 \\
0\end{array}$ & $\begin{array}{l}0 \\
0 \\
0 \\
0 \\
0 \\
0 \\
0 \\
0 \\
0 \\
0 \\
0 \\
0 \\
0 \\
0 \\
0 \\
0 \\
0 \\
0 \\
0 \\
0\end{array}$ & $\begin{array}{l}0 \\
0 \\
0 \\
0 \\
0 \\
0 \\
0 \\
0 \\
0 \\
0 \\
0 \\
0 \\
0 \\
0 \\
0 \\
0 \\
0 \\
0 \\
0 \\
0\end{array}$ & $\begin{array}{l}0 \\
0 \\
0 \\
0 \\
0 \\
0 \\
0 \\
0 \\
0 \\
0 \\
0 \\
0 \\
0 \\
0 \\
0 \\
0 \\
0 \\
0 \\
0 \\
0\end{array}$ & $\begin{array}{l}0 \\
0 \\
0 \\
0 \\
0 \\
0 \\
0 \\
0 \\
0 \\
0 \\
0 \\
0 \\
0 \\
0 \\
0 \\
0 \\
0 \\
0 \\
0 \\
0\end{array}$ & $\begin{array}{l}0 \\
0 \\
0 \\
0 \\
0 \\
0 \\
0 \\
0 \\
0 \\
0 \\
0 \\
0 \\
0 \\
0 \\
0 \\
0 \\
0 \\
0 \\
0 \\
0 \\
0 \\
0 \\
0\end{array}$ & $\begin{array}{l}0 \\
0 \\
0 \\
0 \\
0 \\
0 \\
0 \\
0 \\
0 \\
0 \\
0 \\
0 \\
0 \\
0 \\
0 \\
0 \\
0 \\
0 \\
0 \\
0\end{array}$ & $\begin{array}{l}0 \\
0 \\
0 \\
0 \\
0 \\
0 \\
0 \\
0 \\
0 \\
0 \\
0 \\
0 \\
0 \\
0 \\
0 \\
0 \\
0 \\
0 \\
0 \\
0 \\
0\end{array}$ & $\begin{array}{l}0 \\
0 \\
0 \\
0 \\
0 \\
0 \\
0 \\
0 \\
0 \\
0 \\
0\end{array}$ \\
\hline
\end{tabular}




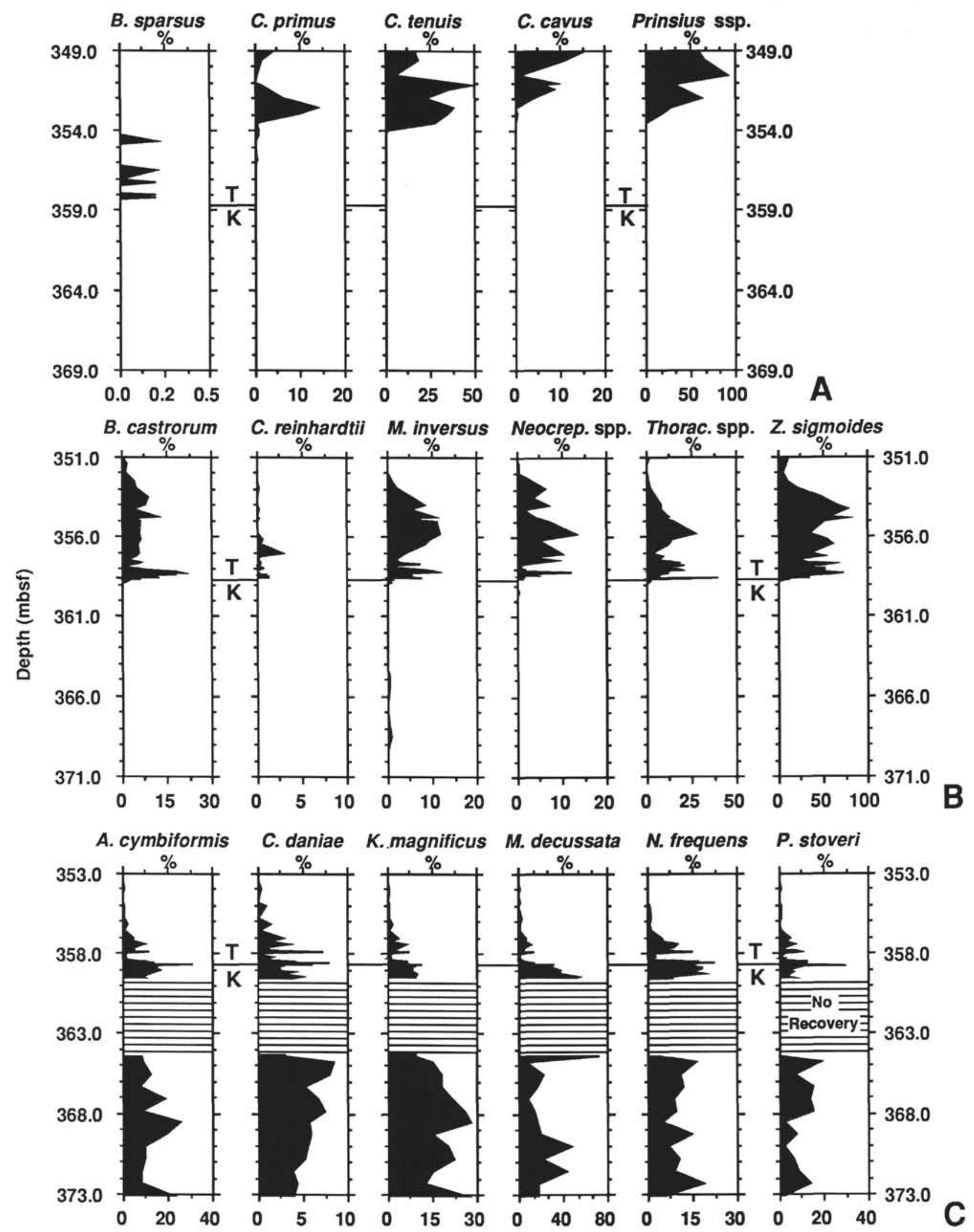

Figure 7. Percent abundance of selected individual nannofossil species across the Cretaceous/Tertiary boundary ODP Hole 752B. A. Tertiary taxa. B. "Survivor" taxa. C. Cretaceous taxa. Note the scale change for individual species percent abundance (horizontal axis). 
The abundance of Cretaceous species drops to $4 \%$ in Sample 121-752B-11R-3, 44-45. cm, $48 \mathrm{~cm}$ above the boundary (Fig. 6, Table 1). Above this, in Sample 121-752B-11R-3, 12-13 cm, an interval of intense reworking is present as Cretaceous species constitute $93 \%$ of the assemblage. Reworking is prevalent, although not as intense up through Sample 121-752B-11R-2, 70-71 $\mathrm{cm}$. The sharp peaks in Cretaceous species abundance above the boundary are illustrated in Figures 6 and 7. Cretaceous nannofossils were not the only constituents of the sediment redeposited in during this interval of reworking. An additional peak in iridium abundance was noted by Asaro et al. (this volume) coincident with the reworked nannofossils in Sample 121-752B-11R-3, 12-13 $\mathrm{cm}$.

Overall, Cretaceous nannofossils are abundant but preservation is poor, which is reflected in the low diversity and the dominance of dissolution-resistant forms such as Micula decussata, Arkhangelskiella cymbiformis, and Kamptnerius magnificus (Fig. 7). Within the boundary interval, Sample 121-752B-11R-3, 91-92 cm, shows better preservation and the diversity of Cretaceous forms is much higher. The assemblage at this level is dominated by the small, delicate species Prediscosphaera stoveri, which constitutes $30 \%$. The abundance of this form does appear to be related to preservation in this section as only one specimen was recorded in Sample 121-752B-11R-3, 88-89 cm, only $3 \mathrm{~cm}$ above, which contains very rare, poorly preserved nannofossils.

Below the boundary, in Samples 121-752B-11R-3, 101-102 $\mathrm{cm}$, to $121-752 \mathrm{~B}-12 \mathrm{R}-6,115-116 \mathrm{~cm}$, the dominant Cretaceous form is $M$. decussata. This species comprises a maximum of $71 \%$ of the assemblage in Sample 121-752B-11R-CC (Fig. 7 and Table 1) and averages around in $30 \%$ in the Maestrichtian samples examined. Thierstein (1981) reported abundances averaging between $40 \%$ and $50 \%$ for this form at DSDP Site 356 (western South Atlantic) and at the Braggs, Alabama, section, a prominent peak of about $50 \%$ was noted just below the Cretaceous/Tertiary boundary. These abundance peaks were attributed to poor preservation. Results from experiments on nannofossil preservation by Thierstein (1980) have shown that $M$. decussata is a highly dissolution-resistant form susceptible to diagenetic overgrowth. The abundance of this taxa is enhanced in sediments where dissolution and mobilization of calcite has taken place. In the present section, where preservation of nannofossils is poor, $M$. decussata is strongly overgrown and hence, its abundance is probably enhanced by the dissolution of more delicate species. Likewise, although noted to be more abundant in the high latitude sections, the percentages of robust forms such A. cymbiformis and $K$. magnificus may also be further enhanced by this process.

Dissolution may not be the sole factor controlling the abundance of forms such as $M$. decussata. In the uppermost Maestrichtian portion of a Cretaceous/Tertiary section in Israel, Moshkovitz and Eshet (1989) reported very abundant $M$. decussata in samples where preservation was good and there was no evidence of strong dissolution or overgrowth. Hence, elevated abundances of $M$. decussata in uppermost Maestrichtian sediments in sections elsewhere could also reflect a natural increase in abundance of this species in addition to diagenetic enhancement.

The other major components of the Cretaceous assemblage include, A. cymbiformis, K. magnificus, Nephrolithus frequens frequens, Prediscosphaera cretacea, $P$. stoveri, and Cribrosphaerella daniae. This assemblage lacks middle to low latitude components such as $M$. murus, $M$. prinsii, and common to abundant Watznaueria barnesae, and is characteristic of the high latitudes for the end of the Maestrichtian (Thierstein, 1981; Pospichal and Wise, 1990a, 1990b). The abundance of $P$. stoveri is much lower at this site than at Site 690 on Maud Rise $\left(65^{\circ} \mathrm{S}\right)$ and at Site $738\left(63^{\circ} \mathrm{S}\right)$. This difference is attributed, in part, to poorer preservation, and possibly to the more northerly location of Site $752\left(50^{\circ}-55^{\circ} \mathrm{S}\right)$. Also, it may be important to note that it is not known what the abundance of this form is in the unrecovered sediments comprising the 5-m gap in Core 121-752B-11R. It is entirely possible that this species may have peaked at much higher abundances in this 5-m interval.

The abundance of $P$. stoveri reaches nearly $70 \%$ below the Cretaceous/Tertiary boundary at Site 690 (Pospichal and Wise, 1990a) and possibly just as high at Site 738. It appears that the late Maestrichtian bloom of $P$. stoveri was not as intense at middle and lower latitudes. Jiang and Gartner (1986) reported an abundance of $10 \%-15 \%$ for Prediscosphaera quadripunctata, a possible ecophenotypical variation (S. Gartner, pers. comm., 1989), in the uppermost Maestrichtian of the lower latitude sections of Brazos River, Texas, and El Kef, Tunisia (see discussion in Pospichal and Wise, 1990a).

\section{Survivors}

"Survivor" forms are very rare below the Cretaceous/Tertiary boundary and generally amount to less than $1 \%$ of the total assemblage. Markalius inversus, Zygodiscus sigmoides, Thoracosphaera, and Braarudosphaera are "survivor" forms most likely to be observed in Upper Cretaceous sediments. At this site, very rare $M$. inversus and $Z$. sigmoides are present below the boundary. "Survivor" forms begin to appear with consistency at about $92-93 \mathrm{~cm}$ in Section 121-752B-11R-3. The abundance at this level is about $5 \%$ and rapidly increases to $90 \%$ at $60-61 \mathrm{~cm}$ and peaks at $97 \%$ just below the CP1a/1b boundary in Sample 121-752B-10R-7, 59-56 cm, or about $4 \mathrm{~m}$ above the boundary.

Other than those mentioned previously, the "survivor" assemblage on Broken Ridge includes Biscutum castrorum, Cyclagelosphaera reinhardtii, C. alta, Lapideacassis sp., Markalius apertus, Neocrepidolithus cruciatus, N. fossus (Pl. 1, Fig. 10), and $N$. neocrassus. Included in this assemblage are small $(4-5 \mu \mathrm{m})$ oval forms with a central cross structure either aligned with the major and minor axes or slightly rotated (Pl. 1, Figs. 11 and 12). These forms, referred to here as "Zygolithus crux," may be synonymous with or closely related to the Danian species, Chiastozygus ultimus described by Perch-Nielsen (1981a).

The most abundant "survivor" form is Zygodiscus sigmoides, which ranges up to $75 \%$ of the assemblage within Subzone CP1a (Fig. 7). The acme of this form is noted in several sections and is most pronounced in higher latitudes (Perch-Nielsen, 1979a, $1979 b)$. At Site 690 in the Weddell Sea, Z. sigmoides is also the dominant "survivor" form, but there its peak abundance does not exceed 40\% (Pospichal and Wise, 1990a).

Thoracosphaera attain the next highest abundance levels within the "survivor" assemblage. This form reaches a peak abundance of about $25 \% 3 \mathrm{~m}$ above the boundary and averages around $10 \%-15 \%$ within Subzone CP1a (Fig. 7). In Sample 121-752B$11 \mathrm{R}-3,80-81 \mathrm{~cm}$ (358.60 mbsf), two Thoracosphaera were noted in a count of five nannofossils that accounts for the pronounced thin peak above the boundary shown in Figure 7. Nannofossils are rare in the cherty sediments of this interval and preservation is poor, thus the abundance data, although plotted, are not considered a true reflection of the assemblage composition.

The abundance of Thoracosphaera appears to vary in sections from around the globe. Although blooms of Thoracosphaera were reported by Perch-Nielsen et al. (1982) to have a worldwide distribution, this was later found not to be the case at Site 690 in the Weddell Sea (Pospichal and Wise, 1990a). At this site, the abundance of Thoracosphaera is no more than 5\%-6\% above the boundary and does not increase until the zones above. This is in contrast to the peak abundances of $50 \%$ or so found at Site 524 in the South Atlantic (Perch-Nielsen et al., 1982) and the high abundances noted in the marginal Tethyan sequences. A similar 
pattern is noted for Braarudosphaera, of which only six scattered specimens were observed here.

The percent abundances of Biscutum castrorum, Markalius inversus, and Neocrepidolithus spp. all range about $10 \%$ or less immediately above the boundary in Subzone CP1a. Cyclagelosphaera, Lapideacassis sp., Braarudosphaera biglowii, and " $\mathrm{Zy}$ golithus crux" generally make up $1 \%-2 \%$ of the assemblage. The abundance patterns of these species are similar to those found at Site 690 , but in general, slightly higher percentages occur at Site 752 where reworking of Cretaceous species is not as pervasive as at Site 690 . The higher percentage of reworked Cretaceous forms at Site 690 probably lowers the apparent abundances of "survivor" taxa. For this reason, in future studies it may be more accurate to compare components of the "survivor" assemblage independent of percentages of reworked Cretaceous species.

\section{Tertiary}

The first Tertiary species to appear is Biantholithus sparsus in Sample 121-752B-11R-3, 72-73 cm (358.52 mbsf). This taxon is very rare, never more than one specimen per count of 500. Its first occurrence was used by Perch-Nielsen (1979a) to denote the boundary and was also effectively used to mark the boundary at Site 690 (Pospichal and Wise, 1990a). However, its occurrence is rare and sporadic and may not be the best criteria to delimit the Cretaceous/Tertiary horizon in all sections.

The next forms to appear above the boundary are small oval placoliths that may be Cruciplacolithus primus with the central cross structure dissolved out (Pl. 1, Figs. 1-3). These forms are rare, starting at Sample 121-752B-11R-3, 39-40 cm (358.19 mbsf), $73 \mathrm{~cm}$ above the boundary. At first it was thought that they may be Neobiscutum romeinii (Pospichal in Peirce, Weissel, et al., 1989, p. 512), but this could not be confirmed by scanning electron microscopy. Thus, because of uncertainties in identification, they are placed in the category of "miscellaneous small oval placoliths" in Table 1. Cruciplacolithus primus, less than $5 \mu \mathrm{m}$ in length and with a visible cross structure (Pl. 1, Fig. 6) first appear in Sample 121-752B-11R-1, 98-99 cm (355.78 mbsf), about 2.5 $\mathrm{m}$ above the small oval forms. This taxon is rare at first but peaks at $10 \%$ of the assemblage at Sample 121-752B-10R-5, 143-144 $\mathrm{cm}$ (352.53 mbsf). The highest abundance of "miscellaneous placoliths" also occurs in this sample, suggesting that the two are probably the same form. Just below the $\mathrm{CP} 1 \mathrm{a} / 1 \mathrm{~b}$ boundary in Sample 121-752B-10R-7, 17-18 cm $(354.27 \mathrm{~cm})$, a larger variant of $C$. primus appears. These specimens are between 5 and $7 \mu \mathrm{m}$ long, have a more robust central area than the smaller $C$. primus, and are morphologically more similar to $C$. tenuis, which appears in the sample above. The graphic abundance of $C$. primus shown in Figure 7 includes both small and larger varieties and the "miscellaneous placoliths." The combined group of $C$. primus reaches a maximum abundance of about $15 \%$ slightly above the CP1a/1b boundary, but its numbers are generally much less as $C$. tenuis and Prinsius tenuiculum/dimorphosus completely dominate the assemblage.

Specimens of the Cruciplacolithus lineage greater than $7 \mu \mathrm{m}$ are considered to be $C$. tenuis (Pl. 1, Figs. 4, 5). This same taxonomic concept was used for Site 690 and is discussed further in Pospichal and Wise (1990a). The first occurrence of this species in Sample 121-752B-10R-6, 135-136 cm, (353.95 mbsf) is used to mark the $\mathrm{CP} 1 \mathrm{a} / 1 \mathrm{~b}$ zonal boundary. Its abundance ranges from $25 \%$ to $50 \%$ of the assemblage but it is rapidly replaced as the most abundant species by Prinsius spp. in Sample 121-752B10R-4, 95-96 cm (350.55 mbsf).

Minute specimens of Prinsius probably belonging to $P$. tenuiculum (Pl. 1, Figs. 8, 9) first occur in Sample 121-752B$10 \mathrm{R}-6,86-87 \mathrm{~cm}$, which is above the FO of $C$. tenuis. These forms are as small as $1.5 \mu \mathrm{m}$ and are difficult to detect in the light microscope, thus their actual first occurrence may be below what is recorded here. They are generally not noticed until they become abundant, which they do quite rapidly. The smaller forms are joined in Sample 121-752B-10R-4, 95-96 cm, by larger forms (3-4 $\mu \mathrm{m})(P$. dimorphosus) and the abundances range about $60 \%-80 \%$ (Fig. 7). Large abundances of Prinsius are also noted at Site 690 (Pospichal and Wise, 1990c) and in sections from the North Sea and Denmark (Perch-Nielsen, 1979a).

Coccolithus cavus first appears in Sample 121-752B-10R-6, $32-33 \mathrm{~cm}$, and attains abundances ranging from $5 \%$ to $15 \%$. Futyania petalosa is also present in the section, however, only one specimen was observed in Sample 121-752B-10R-5, 143-144 $\mathrm{cm}$ (Pl. 1, Fig. 13). When rare, this form also is difficult to detect in the light microscope, thus its abundance may be higher than recorded here. Observations of samples from Site 750 revealed several specimens of $F$. petalosa from the same stratigraphic level. This site lies to the south of the paleoposition of Site 752 (Fig. 1). No specimens were observed at Site 690. Looking at the distribution of $F$. petalosus on a global scale, it appears to prefer lower to middle latitudes with some blooms in these regions (Perch-Nielsen et al., 1982).

One component of the early Tertiary assemblage that is absent at Site 752 but quite conspicuous at Sites 690 and 738 is Hornibrookina, which blooms above the Cretaceous/Tertiary horizon near the CP1a/1b boundary. This was first noted from Site 690 (Pospichal and Wise, 1990a) where these forms, referred to as $H$. edwardsii, reached abundances of up to $50 \%$ of the assemblage. In the present site, Hornibrookina (Pl. 1, Fig. 7) does not appear until higher in the section, just below Zone CP2, where one specimen was observed after the count of 500 in Sample 121752B-10R-1, 96-97 cm. Hornibrookina was noted at nearby Site 750 within Zone CP1b and probably extends down near the base of this zone (CP1 a is absent at this site). At the higher latitude Site 738 , Hornibrookina first occurs about $15 \mathrm{~cm}$ above the boundary and has abundances similar to those at Site 690 (Wei and Pospichal, in press). The lack of a bloom at Site 752 confines this peculiar event to the extreme high austral latitudes. Although Hornibrookina is known from boreal sections (Jiang and Gartner, 1986), no blooms have been recorded there. The northern limits of this bloom in the Indian Ocean apparently lie somewhere between Sites 750 and 752 , which would have been separated at that time by no more than an estimated $10^{\circ}$ of latitude and probably much less. Hornibrookina is discussed in more detail in Pospichal and Wise (1990a) and Wei and Pospichal (in press).

\section{SUMMARY AND CONCLUSIONS}

Combined biostratigraphic, magnetostratigraphic, and geochemical analyses confirm that the Cretaceous/Tertiary boundary recovered in ODP Hole 752B is essentially complete. The boundary lies well within Chron $29 \mathrm{R}$ (Gee et al., this volume) and contains anomalously high abundances of the trace element, iridium (Asaro et al, this volume).

Sediment-accumulation rates across the boundary dropped dramatically as productivity of the calcareous microplankton declined. However, a constant supply of volcanic ash slightly offset this decline in biogenic sedimentation to produce a fairly thick boundary section. The lowermost subzone, CP1a, is nearly $5 \mathrm{~m}$ thick, which corresponds to the time of suppressed surface productivity. The duration of this subzone is about $300,000 \mathrm{yr}$ according to Berggren et al. (1985). If the amount $\mathrm{CaCO}_{3}$ accumulation is taken as a measure of productivity, however, then the period of low production extends beyond $300,000 \mathrm{yr}$ and is probably between $500,000 \mathrm{yr}$ and $1.5 \mathrm{~m}$.y. This duration is consistent with carbon isotope analyses of deep-sea Cretaceous/Tertiary sections by Zachos et al. (1989), but longer than that proposed by Stott and Kennett (1989) for the section at Site 690. 


\section{J. J. POSPICHAL}

The section at Site 752 is much more expanded than other DSDP/ODP deep-sea sections recovered. Figure 8 shows a comparison of the thicknesses of Subzone CP1a from various localities around the globe, including some land-based sequences. Marginal sections such as the one at El Kef, Tunisia, which is used as a standard for comparison, are generally more expanded as sedimentation rates are higher near shore. The lowest sedimentation rates, and hence the thinnest sequences, are found in the deep sea. The deep-sea section at Site 752 thus provides a better comparison to these expanded sequences because thicknesses exceed the limits of bioturbation, which can obscure biostratigraphic events in sections where the uppermost Maestrichtian or lowermost Danian biozones are less than $1 \mathrm{~m}$ thick.

Calcareous nannofossil assemblages of the uppermost Maestrichtian are comparable to austral high-latitude sections such as those recovered from Site 690 in the Weddell Sea, Antarctica, and southern Kerguelen Plateau Sites 738 and 750. Prediscosphaera stoveri, which completely dominates the uppermost Cretaceous assemblage at Site 690,738 , and 750 , is not as abundant at Site 752 due partly to poor preservation, but also due, apparently, to the lower latitudinal position of this site. The dissolution-resistant species, Micula decussata is dominant this site along with the high latitude forms, Kamptnerius magnificus, Arkhangelskiella cymbiformis, and Nephrolithus frequens. Latest Maestrichtian species which prefer lower latitudes such as Micula murus and $M$. prinsii are not present at this site.

Components of the "survivor" assemblage at Site 752 rapidly replace Cretaceous forms beginning at the Cretaceous/Tertiary boundary and continuing through Subzone CP1a. Zygodiscus sigmoides is the most abundant "survivor" species and is accompanied by Thoracosphaera, Markalius inversus, Biscutum castrorum, and Neocrepidolithus. No Braarudosphaera bloom is present at this site as noted in some other Cretaceous/Tertiary sections.

The newly evolved Tertiary species include Biantholithus sparsus followed by small forms probably best identified as Cruciplacolithus primus minus the central cross. C. tenuis, Prinsius spp., and Coccolithus cavus dominate the Subzone CP1b assemblage. Hornibrookina is conspicuously absent from this assemblage, which may be attributed to the more northerly location of Site 752 in comparison with Sites 690, 738, and 750. On the other hand, the Tertiary assemblage of the present section lacks certain components commonly reported from lower latitude sections such as Brazos River (Jiang and Gartner, 1986) and El Kef (Perch-Nielsen, 1981b). Of these, only one specimen of Futyania petalosa was observed and Neobiscutum romeinii could not be positively identified. In addition, $N$. parvulum was not noted.

In conclusion, the calcareous nannofossil assemblages of the uppermost Maestrichtian and lowermost Paleocene assemblages of Site 752 are similar to those reported from the high latitude localities of the Kerguelen Plateau, Sites 738 and 750 of the southern Indian Ocean, and Weddell Sea, Site 690. However, the lack of a Hornibrookina bloom or a strong influx of Prediscosphaera stoveri at Site 752 may suggest a strong bioprovinciality of these events at least across the Kerguelen Plateau. This is best illustrated by Hornibrookina, which is abundant in sediments of CP1b at Site $750\left(57^{\circ} \mathrm{S}\right)$ but absent at this level at Site 752 (estimated $50^{\circ}-55^{\circ} \mathrm{S}$ ). Allowing for plate movement, these two sites would have been in close proximity at this time, separated by a maximum of $10^{\circ}$ latitude and probably less (Fig. 1B). Thus, for at least the Indian Ocean, Site 752 may represent the northern limit for the biogeographic province indicated by the assemblages described from Sites 690, 738, and 750 .

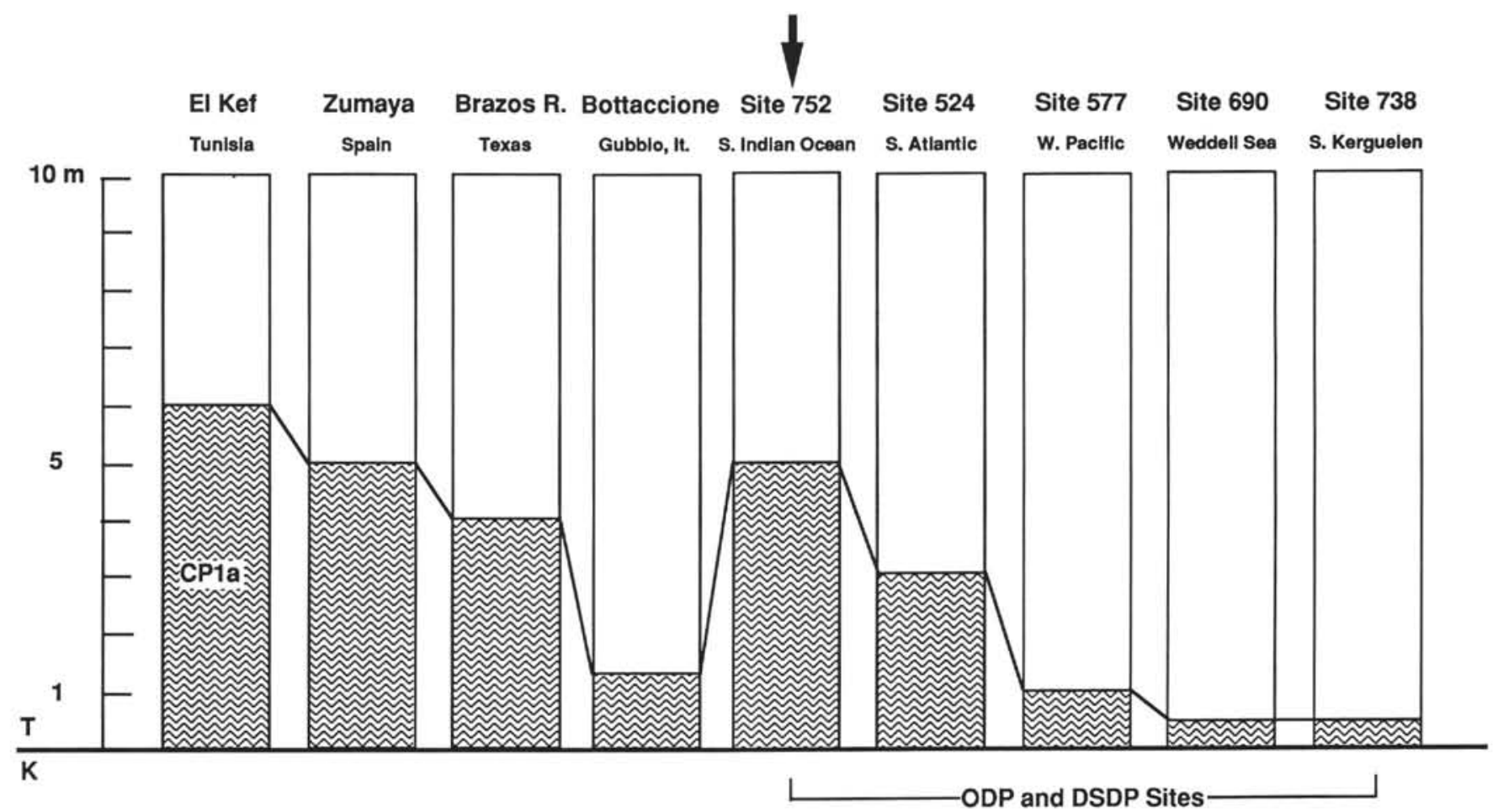

Figure 8. Comparison of the thickness of Subzone CP1 a (NP1) from various land-based and DSDP/ODP Cretaceous/Tertiary sections (after Pospichal and Wise, 1990a). Thicknesses are implied for sections in which the standard zonation schemes were not applied. El Kef (Perch-Nielsen, 1981b); Zumaya (Percival and Fischer, 1977); Brazos River (Jiang and Gartner, 1986); Bottaccione (Monechi and Thierstein, 1985); Site 524 (Percival, 1984); Site 577 (Monechi, 1985); Site 690 (Pospichal and Wise, 1990a); Site 738 (Wei and Thierstein, in press). 


\section{ACKNOWLEDGMENTS}

This study was supported by Leg 121 USSAC funds, National Science Foundation grant DDP-8917976, an equipment grant from the Amoco Foundation, and an Aylesworth Foundation Fellowship. Special thanks goes to Dr. S. W. Wise for critical review of this manuscript and for overall guidance. Suggestions by Dr. Katharina von Salis Perch-Nielsen and one anonymous reviewer greatly improved this manuscript. I also thank my shipmates for many helpful discussions and for making Leg 121 a truly enjoyable adventure.

\section{REFERENCES}

Berggren, W. A., Kent, D. V., Flynn, J. J., and Van Couvering, J. A., 1985. Cenozoic geochronology. Geol.Soc. Am. Bull., 96:1407-1418.

Bramlette, M. N., and Martini, E., 1964. The great change in calcareous nannoplankton fossils between the Maestrichtian and Danian. Micropaleontology, 10:291-322.

Edwards, A. R., 1966. Calcareous nannoplankton from the uppermost Cretaceous and the lowermost Tertiary of the Mid-Waipara section, South Island, New Zealand. N. Z. J. Geol. Geophys., 9:481-490.

1973. Calcareous nannofossils from the southwest Pacific, Deep Sea Drilling Project, Leg 21. In Burns, R. E., Andrews, J. E., et al., Init. Repts. DSDP, 21: Washington (U.S. Govt. Printing Office), 641-691.

Ehrendorfer, T., and Aubry, M. P., in press. Calcareous nannoplankton changes across the Cretaceous/Paleocene boundary in the Southern Indian Ocean (ODP Site 750A). In Schlich, R., Wise, S. W., Jr., et al., Proc. ODP, Sci. Results, 120: College Station, TX (Ocean Drilling Program).

Hay, W. W., and Mohler, H. P., 1967. Calcareous nannoplankton from early Tertiary rocks at Pont Labau, France, and Paleocene-Eocene correlations. J. Paleontol., 41:1505-1541.

Jiang, M. J., and Gartner, S., 1986. Calcareous nannofossil succession across the Cretaceous/Tertiary boundary in east-central Texas. Micropaleontology, 32:232-255.

Martini, E., 1971. Standard Tertiary and Quaternary calcareous nannoplankton zonation. In Farinacci, A. (Ed.), Proc. 2nd Planktonic Conf. Roma: Rome (Ed. Technosci.), 2:739-785.

Monechi, S., 1977. Upper Cretaceous and early Tertiary nannoplankton from the Scaglia Umbra Formation (Gubbio, Italy). Riv. Ital. Paleontol., 83:759-802.

1985. Campanian to Pleistocene calcareous nannofossil stratigraphy from the northwest Pascific Ocean, Deep Sea Drilling Project Leg 86. In Heath, G. R., Burckle, L. H., et al. Init. Repts. DSDP, 86: Washington (U.S. Govt. Printing Office), 301-336.

Monechi, S., and Thierstein, H. R., 1985. Late Cretaceous-Eocene nannofossil and magnetostratigraphic correlations near Gubbio, Italy. Mar. Micropaleontol., 9:419-440.

Moshkovitz, S., 1978. New types of cover-slip and mounting-slide with a graticule for examinations of the same small object both by the light microscope and the scanning electron microscope. Microscop. Acta, 80:161-166.

Moshkovitz, S., and Eshet, Y., 1989. Inter-relative aspects of nannofossil and palynomorph biostratigraphy and paleoecology at the K/T boundary, Hor Ha'ar section, southern, Israel. INA Newsl., 11:81-82.

Okada, H., and Bukry, D., 1980. Supplementary modification and introduction of code numbers to the low latitude coccolith biostratigraphic zonation (Bukry, 1973; 1975). Mar. Micropaleontol., 5:321-325.

Peirce, J., Weissel, J., et al., 1989. Proc. ODP, Init. Repts., 121: College Station, TX (Ocean Drilling Program).

Perch-Nielsen, K., 1969. Die Coccolithen einger danischer Maastrichtien und Danienlokalitaten. Bull. Geol. Soc. Den., 19:51-66.

1979a. Calcareous nannofossil zonation at the Cretaceous/Tertiary boundary in Denmark. In Birkelund, T., and Bromley, R. G. (Eds.), Cretaceous-Tertiary Boundary Events (Vol. 1). Copenhagen (Univ. of Copenhagen), 115-135.

1979b. Calcareous nannofossils in Cretaceous/Tertiary boundary sections in Denmark. In Christensen, W. K. and Birkelund, T. (Eds.), Cretaceous-Tertiary Boundary Events (Vol. 2). Copenhagen (Univ, of Copenhagen), 120-126. 1981a. New Maastrichtian and Paleocene calcareous nannofossils from Africa, Denmark, the USA and the Atlantic, and some Paleocene lineages. Eclogae Geol. Helv., 74:831-863.

$1981 \mathrm{~b}$. Nouvelles observations sur les nannofossiles calcaires à la limite Crétacé-Tertiaire près de El Kef (Tunisie). Cah. Micropaleont., 3:25-36.

Perch-Nielsen, K., McKenzie, J. A., and He, Q., 1982. Biostratigraphy and isotope stratigraphy and the "catastrophic" extinction of calcareous nannoplankton at the Cretaceous/Tertiary boundary. Spec. Pap. Geol. Soc. Am., 190:353-371.

Percival, S. F., Jr., 1984. Late Cretaceous to Pleistocene calcareous nannofossils from the South Atlantic, Deep Sea Drilling Project Leg 73. In Hsü, K. J., LaBrecque, J. L., et al., Init. Repts. DSDP, 73: Washington (U.S. Govt. Printing Office), 391-424.

Percival, S. F., Jr., and Fisher, A. G., 1977. Changes in calcareous nannoplankton in the Cretaceous-Tertiary biotic crisis at Zumaya, Spain. Evol. Theory, 2:1-35.

Pospichal, J. J., Wise, S. W., Asaro, F., and Hamilton, N., 1990. The effects of bioturbation across a biostratigraphically complete, high southern latitude K/T boundary. In Sharpton, V., and Ward, P. (Eds.), Proceeding of the Conference on Global Catastrophes in Earth History: An Interdisciplinary Conference on Impacts, Volcanism, and Mass Mortality. Spec. Pap. Geol. Soc. Am., 247:497-508.

Pospichal, J. J., and Wise, S. W., Jr., 1990a. Calcareous nannofossils across the K/T boundary, ODP Hole 690C, Maud Rise, Weddell Sea. In Barker, P. F., Kennett, J. P., et al., Proc. ODP, Sci. Results, 113: College Station, TX (Ocean Drilling Program), 515-532.

$1990 \mathrm{~b}$. Maestrichtian calcareous nannofossil biostratigraphy of Maud Rise ODP Leg 113 Sites 689 and 690, Weddell Sea. In Barker, P. F., Kennett, J. P., et al., Proc. ODP, Sci. Results, 113: College Station, TX (Ocean Drilling Program), 465-487.

1990c. Paleocene to middle Eocene calcareous nannofossils of ODP Sites 689 and 690, Maud Rise, Weddell Sea. In Barker, P. F., Kennett, J. P., et al., Proc. ODP, Sci. Results, 113: College Station, TX (Ocean Drilling Program), 613-638.

Rea, D. K., Dehn, J., Driscoll, N., Farrell, J., Janecek, T., Owen, R. M., Pospichal, J. L., Resiwati, P., and the ODP Leg 121 Scientific Party, 1990. Paleoceanography of the Eastern Indian Ocean from ODP Leg 121 Drilling on Broken Ridge. Geol. Soc. Am. Bull., 102:679-690.

Romein, A.J.T., 1977. Calcareous nannofossils from the Cretaceous/Tertiary boundary interval in the Barranco del Gredero (Caravaca, Prov. Murcia, S. E. Spain), I and II. Proc. K. Ned. Akad. Wet., Ser. B: Palaeontol., Geol., Phys., Chem., 80:256-279.

Schlich, R., Wise, S. W., Jr., et al., 1989. Proc. ODP, Init Repts., 120: College Station, TX (Ocean Drilling Program).

Sissingh, W., 1977. Biostratigraphy of Cretaceous calcareous nannoplankton. Geol. Mijnbouw, 56:37-65.

Smit, J., and Romein, A.J.T., 1985. A sequence of events across the Cretaceous-Tertiary boundary. Earth Planet. Sci. Lett., 74:155-70.

Stott, L. D., and Kennett, J. P., 1989. New constraints on early Tertiary palaeoproductivity from carbon isotopes in foraminifera. Nature, 342:526-529.

Thierstein, H. R., 1980. Selective dissolution of late Cretaceous and earliest Tertiary calcareous nannofossils: experimental evidence. Cretaceous Res., 2:165-176.

1981. Late Cretaceous nannoplankton and the change at the Cretaceous-Tertiary boundary. In Warme, J. E., Douglas, R. G., Winterer, E. L. (Eds.), The Deep Sea Drilling Project: A Decade of Progress: Soc. Econ. Paleontol. Mineral., Spec. Publ., 32:355-394.

Thierstein, H. R., Asaro, F., Ehrmann, W. U., Huber, B., Michel, H., Sakai, H., Schmitz, B., in press. The Cretaceous-Tertiary boundary at Site 738, south Kerguelen Plateau. In Barron, J., Larsen, B., et al., Proc. ODP, Sci. Results, 119: College Station, TX (Ocean Drilling Program).

Thierstein, H. R., and Okada, H., 1979. The Cretaceous/Tertiary boundary event in the North Atlantic. In Tucholke, B. E., Vogt, P. R., et al., Init. Repts. DSDP, 43: Washington (U.S. Govt. Printing Office), 601-616. van Heck, S. E., and Prins, B., 1987. A refined nannoplankton zonation for the Danian of the Central North Sea. Abh. Geol. Bundensanst. Austria, 39:285-303.

Watkins, D. K., in press. Upper Cretaceous nannofossils form ODP Leg 120, Kerguelen Plateau, Southern Ocean. In Schlich, R., Wise, S. W., Jr., et al., Proc. ODP, Sci. Results, 120: College Station, TX (Ocean Drilling Program). 


\section{J. J. POSPICHAL}

Wei, W., and Pospichal, J., in press. Danian calcareous nannofossil succession at ODP Site 7338 in the southern Indian Ocean. In Barron, J., Larsen, B., et al., Proc. ODP, Sci. Results, 119: College Station, TX (Ocean Drilling Program).

Wei, W., and Thierstein, H. R., in press. Upper Cretaceous and Cenozoic calcareous nannofossils of the Kerguelen Plateau (southern Indian Ocean) and Prydz Bay (East Antarctica). In Barron, J., Larsen, B., et al.,Proc. ODP, Sci. Results, 119: College Station, TX (Ocean Drilling Program).

Worsley, T. R., 1974. The Cretaceous/Tertiary boundary event in the ocean. In Hay, W. W. (Ed.), Studies in Paleo-oceanography, Soc. Econ. Paleontol. Mineral. Spec. Publ., 20:94-125.

Zachos, J. C., Arthur, M. A., and Dean, W. E., 1989. Geochemical evidence for suppression of pelagic marine productivity at the Cretaceous/Tertiary boundary. Nature, 337:61-64.

\section{Date of initial receipt: 2 March 1990 \\ Date of acceptance: 3 July 1990 \\ Ms 121B-148}

\section{APPENDIX}

Calcareous Nannofossils Considered in This Report in Alphabetical Order of Generic Epithets

Tertiary

Biantholithus sparsus Bramlette and Martini, 1964

Coccolithus cavus (Hay and Mohler) Perch-Nielsen, 1969

C. pelagicus (Wallich) Schiller, 1930

Cruciplacolithus edwardsii Romein, 1979

C. primus Perch-Nielsen, 1977

C. tenuis (Stradner) Hay and Mohler, 1967

Futyania petalosa (Ellis and Lohmann) Varol, 1989

Hornibrookina edwardsii Perch-Nielsen, 1977

H. teuriensis Edwards, 1973a

Neobiscutum romeinii (Perch-Nielsen) Varol, 1989

N. parvulum (Romein) Varol, 1989

Prinsius dimorphosus (Perch-Nielsen) Perch-Nielsen, 1977

P. tenuiculum (Okada and Thierstein) Perch-Nielsen, 1984

\section{"Survivor"}

Biscutum castrorum Black, 1959

Braarudosphaera bigelowii (Gran and Braarud) Deflandre, 1947

Cyclagelosphaera alta Perch-Nielsen, 1979a

C. reinhardtii (Perch-Nielsen) Romein, 1977
Chiastozygus ultimus Perch-Nielsen, 1981a

Lapideacassis sp.

Markalius apertus Perch-Nielsen, 1979a

M. inversus (Deflandre) Bramlette and Martini, 1964

Neocrepidolithus cruciatus (Perch-Nielsen) Perch-Nielsen, 1981a

N. neocrassus (Perch-Nielsen) Romein, 1979

N. fossus Romein, 1979

Thoracosphaera Kamptner, 1927

Zygodiscus sigmoides Bramlette and Sullivan, 1961

\section{Cretaceous}

Acuturris scotus (Risatti) Wind and Wise in Wise and Wind, 1977

Ahmuellerella octoradiata (Gorka) Reinhardt, 1964

Arkhangelskiella cymbiformis Vekshina, 1959

A. specillata Vekshina, 1959

Bidiscus rotatorius Bukry 1969

Biscutum constans (Gorka) Black, 1959

B. notaculum Wind and Wise in Wise and Wind, 1977

Chiastozygus amphipons (Bramlette and Martini) Gartner, 1968

Chiastozygus sp.

Corollithion rhombicus (Stradner and Adanriker) Bukry, 1969

Cretarhabdus conicus Bramlette and Martini, 1964

Cribrosphaerella daniae Perch-Nielsen, 1973

C. ehrenbergii (Arkhangelsky) Deflandre in Piveteau, 1952

Eiffellithus turriseiffelii (Deflandre and Fert) Reinhardt, 1965

Eiffellithus sp.

Gartnerago spp.

Kamptnerius magnificus Deflandre, 1959

Lithraphadites quadratus Bramlette and Martini (1964)

Micula decussata Vekshina, 1959

M. murus (Martini) Bukry, 1973

M. prinsii Perch-Nielsen, 1979a

Nephrolithus corystus Wind, 1983

N. frequens frequens Gorka, 1957

N. frequens miniporus (Reinhardt and Gorka) Pospichal and Wise, 1990b

Phanulithus obscurus (Prins and Sissingh in Sissingh) Wind and Wise in Wise and Wind, 1977

Prediscosphaera cretacea (Arkhangelsky) Gartner, 1968

P. spinosa (Bramlette and Martini) Gartner, 1968

P. stoveri (Perch-Nielsen) Shafik and Stradner, 1971

Reinhardtites levis Prins and Sissingh in Sissingh, 1977

Tranolithus phacelosus Stover, 1966

Watznaueria barnesae (Black in Black and Barnes) Perch-Nielsen, 1968

Zygodiscus compactus Bukry, 1969

Z. diplogrammus (DeFlandre and Fert) Gartner, 1968

Z. spiralis Bramlette and Martini, 1964

Zygolithus crux (Deflandre and Fert) Bramlette and Sullivan, 1961 

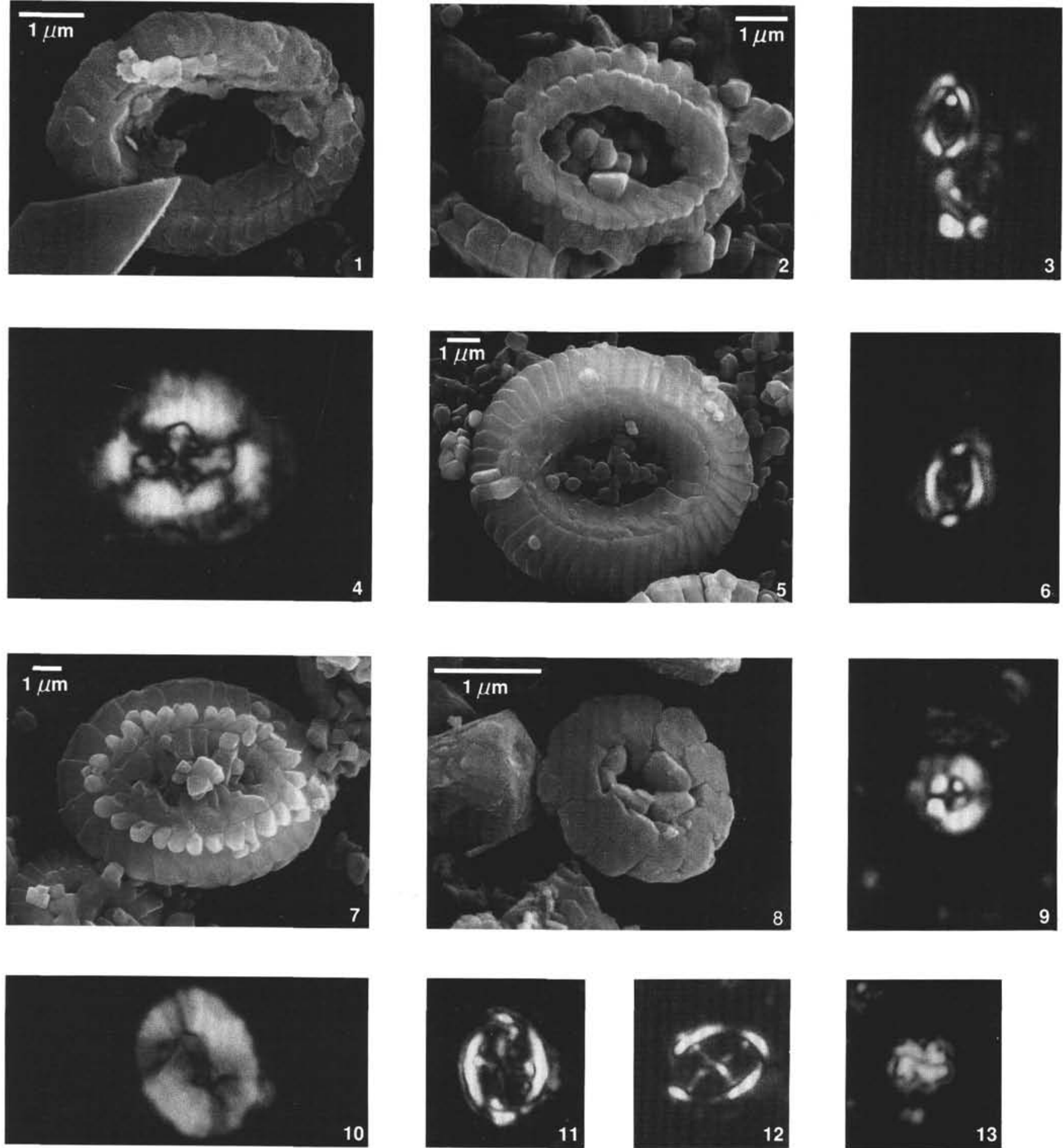

Plate 1. Note on the figures: SEM, Tr, Ph, Pol denote scanning electron micrograph, transmitted, phase contrast, and polarized light, respectively. Magnifications for light micrographs are given in the captions. Scale bars are included on all scanning electron micrographs. All shots are of the distal view except where noted otherwise. 1. Cruciplacolithus primus?, SEM, Sample 121-752B-10R-5, 143-144 cm. 2. C. primus?, SEM, Sample 121-752B-8R-5, 95-96 cm. 3. C. primus?, Pol, $\times 4000$, Sample 121-752B-10R-5, 143-144 cm. 4. C. tenuis, Pol, $\times 4000$, Sample 121-752B-10R-5, 143-144 cm. 5. C. tenuis, SEM, Sample 121-752B-10R-5, 143-144 cm. 6. C. primus, Pol, $\times 4000$, Sample 121-752B-10R-6, 32-33 cm. 7. Hornibrookina teuriensis, SEM, Sample 121-752B-8R-5, 95-96 cm. 8. Prinsius tenuiculum, SEM, Sample 121-752B-10R-5, 143-144 cm. 9. P. tenuiculum, Pol, $\times 4000$, Sample 121-752B-10R-6, 86-87 cm. 10. Neocrepidolithus fossus, Pol, $\times 4000$, Sample 121-752B-10R-5, 143-144 cm. 11. “Zygolithus crux," Pol, $\times 4000$, Sample 121-752B-11R-1, 20-21 cm. 12. "Z. crux," Pol, $\times 4000$, specimen with central bars slightly rotated, Sample $121-752 \mathrm{~B}-11 \mathrm{R}-1,20-21 \mathrm{~cm}$. 13. Futyania petalosa, Pol, $\times 4000$, Sample $121-752 \mathrm{~B}-10 \mathrm{R}-5,143-144 \mathrm{~cm}$. 

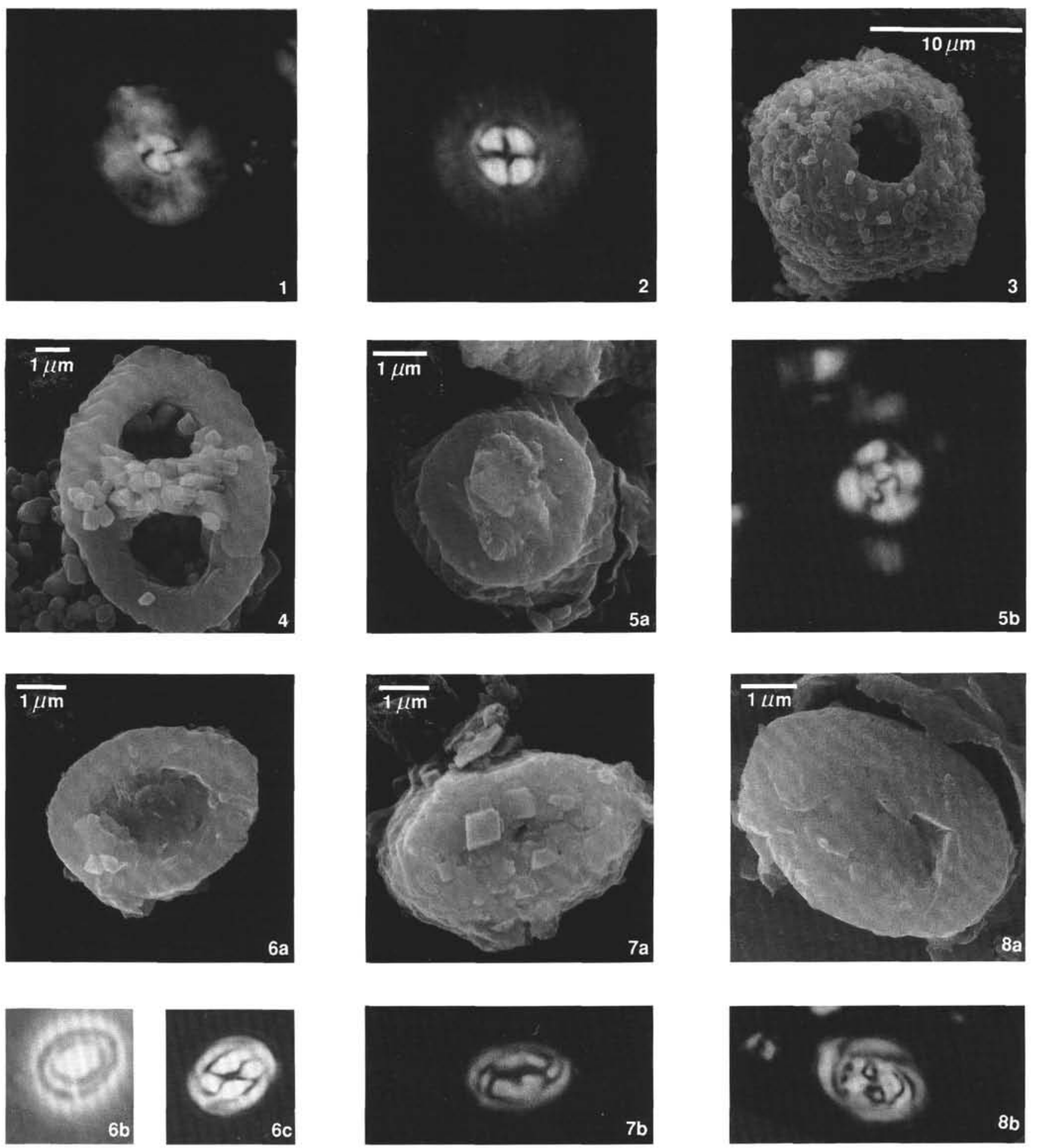

Plate 2. Note: Specimens of Figures 5-8 are photographed in both the light and scanning electron microscope using the correlating technique of Moshkovitz (1978). 1. Biscutum castrorum, Pol, $\times 4000$, Sample 121-752B-10R-5, 143-144 cm. 2. Markalius inversus, Pol, $\times 4000$, Sample 121-752B-11R-1, 20-21 cm. 3. Thoracosphaera operculata, SEM, Sample 121-752B-8R-5, 95-96 cm. 4.Zygodiscus sigmoides, SEM, Sample 121-752B-8R-5, 95-96 cm. 5. A. Cyclagelosphaera reinhardtii, SEM, Sample 121-752B-11R-2, 145-146 cm. B. Same specimen, $\times 4000$, Pol. 6.A. Neocrepidolithus neocrassus, SEM, distal view, Sample 121-752B-1 1R-3, 19-20 cm. B. Same specimen, $\times 4000$, Ph. C. Same specimen, $\times 4000$, Pol. 7. A. N. neocrassus, SEM, proximal view, Sample 121-752B-11R-3, 19-20 cm. B. Same specimen, $\times 4000$, Pol. 8. A. N. cruciatus, SEM, distal view, Sample 121-752B-11R-2, 145-146 cm. B. Same specimen, $\times 4000$, Pol. 

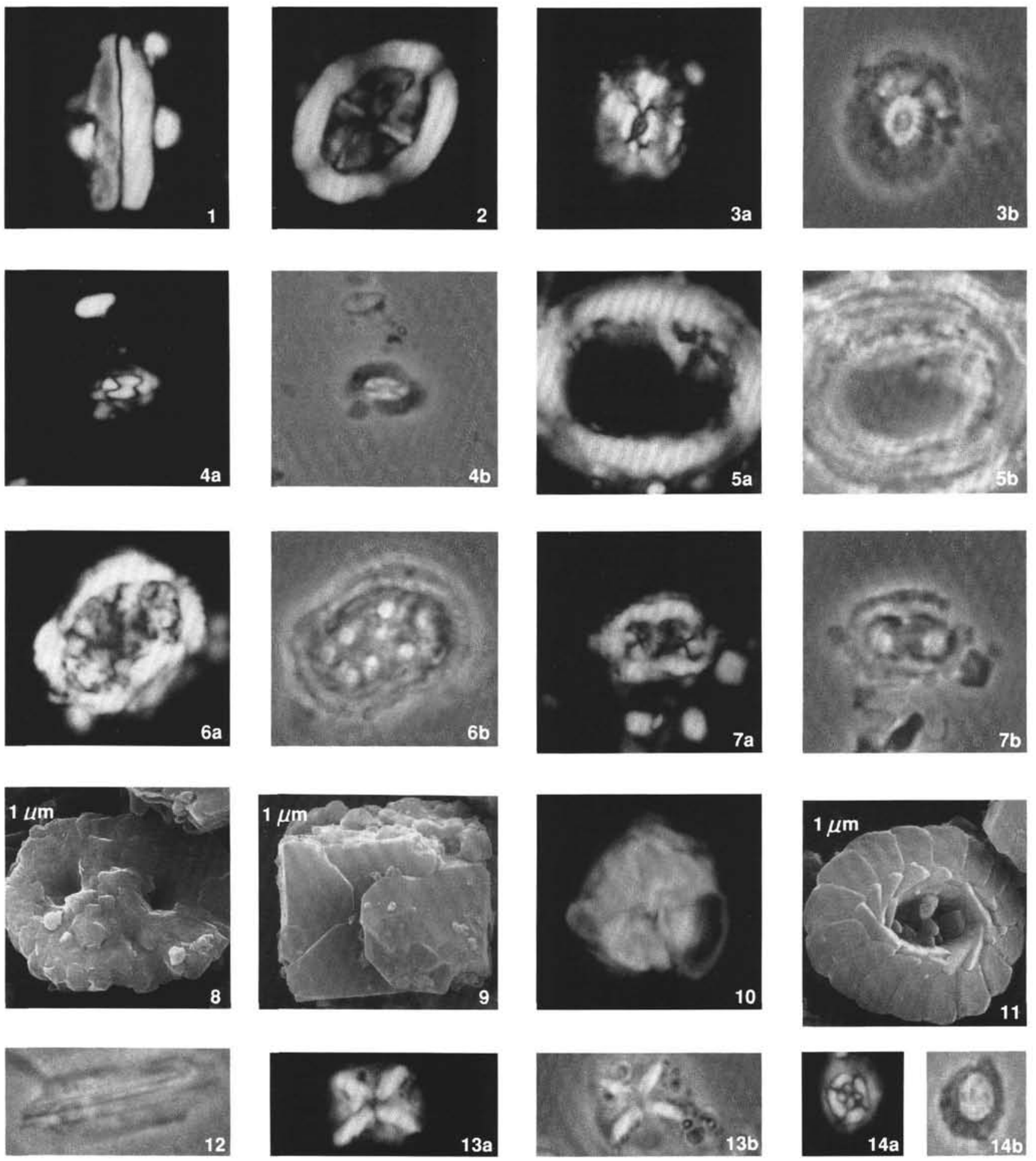

Plate 3. 1. Acuturris scotus, Pol, $\times 3300$, Sample 121-752B-12R-3, 41-42 cm. 2. Arkhangelskiella cymbiformis, Pol, $\times 3300$, Sample 121-752B-12R-3, 41-42 cm. 3. A. Biscutum constans, Pol, $\times 3300$, Sample 121-752B-12R-3, 41-42 cm. B. Ph, $\times 3300$. 4. A. Biscutum sp., Pol, $\times 3300$, Sample $121-752 B-12 R-2,4-41 \mathrm{~cm}$. B. $\mathrm{Ph}, \times 3300$. 5. A. Cribrosphaerella daniae, Pol, $\times 3300$, Sample $121-752 \mathrm{~B}-12 \mathrm{R}-\mathrm{CC}$. B. $\mathrm{Ph}, \times 3300$. 6. A. Nephrolithus frequens frequens, Pol, $\times 3300$, Sample 121-752B-12R-3, 41-42 cm. B. Ph, $\times 3300$. 7. A. N. frequens miniporus, Pol, $\times 3300$, Sample $121-752 \mathrm{~B}-1 \mathrm{IR}-3,91-92 \mathrm{~cm}$. B. Ph, $\times 3300.8$. N. frequens miniporus, SEM, Sample 121-752B-12R-6, 41-42 cm. 9. Micula decussata, overgrown specimen, SEM, Sample 121-752B-12R-6, 41-42 cm. 10. M. decussata, overgown specimen, Pol, $\times 3300$, Sample 121-752B-11R-3,91-92 cm. 11. Prediscosphaera stoveri, SEM, Sample 121-752B-12R-6, 41-42 cm. 12. Lithraphidites quadratus, $\mathrm{Ph}, \times 3300$, Sample $121-752 \mathrm{~B}-12 \mathrm{R}-3,41-42 \mathrm{~cm}$. 13. A. M. decussata, well preserved specimen, Pol, $\times 3300$, Sample $121-752 \mathrm{~B}-11 \mathrm{R}-3,12-13 \mathrm{~cm}$. B. Ph, $\times 3300$. 14. A. P. stoveri, Pol, $\times 3300$, Sample 121-752B-11R-3, $91-92 \mathrm{~cm}$. B. $\mathrm{Ph}, \times 3300$. 